\title{
BUCHBESPRECHUNGEN
}

\section{Ian Brownlie}

\section{The Rule of Law in International Affairs}

International Law at the Fiftieth Anniversary of the United Nations

The Hague Academy of International Law

Kluwer Law International, Den Haag, 1998, 242 pp., £ 53.00

The British mastermind of international law has once again written an excellent book that gives a fantastic overview of the chosen topic as well as insight into related issues: Ian Brownlie is an inspiration to all international lawyers, especially to the young ones at The Hague at the brink of their careers.

This volume inaugurates a new series of books that allows those interested in a subject expounded in the lecture hall of the Academy to consult the lectures in a separate publication. Prof. Brownlie's General Course of 1995 - during the session on public international law - entitled "International Law at the 50th Anniversary of the United Nations" is now enhanced by a preface and an index.

The distinguished scholar states that it is a custom to provide a General Course with a theme of particular flavour and the topic: "The Rule of Law in International Affairs" is more than appropriately chosen as it is a question of perennial concern and was a pressing in 1997/98 when published as it is in 2002 when reviewed.

The author begins explaining his own intellectual background, outlook and approach. He calls himself an "objective positivist", and allows insight into his professional ethics.

Divided into 15 chapters - three preliminary ones and twelve main ones - the book offers a selection of important and pressing topics. The function of law in the international community, the formation of general international law and the subjects of international law form the introduction and then he delves into various areas: the mechanisms of public order, the protection of human rights, the concept of state responsibility, the conditions for the making of international law, the peaceful settlements of disputes, remedies for breaches of obligations, control of major natural resource, title to territory, maritime delimitation, the protection of the territorial integrity and the environment of states, the use of force by states and finally the role of the Security Council and the rule of law.

All subjects are eloquently discussed and analysed by an unequalled writer bringing both academic distinction and his vast experience as a practitioner to every line he shares with the reader.

To do a work like this justice in a review is most difficult, to sum up the wealth of thoughts and ideas of a whole life dedicated to international law presented here impossible. In order to benefit from it one has to read and experience it from cover to cover and savour each 
sentence and statement. It may have been written for students but it is a guide at any stage of any lawyer's or academic's life. There are cross-references, English peculiarities, international comparisons, criticism of the views of renowned colleagues, all well-explained, sharp and to the point. This author always looks at all angles of a case, introduces the reader to various other writers, carefully guides, and still allows space for one's own thoughts.

A joy to read.

Dagmar Reimmann, Tong Norton, England

Eric P.J. Myjer (ed.)

Issues of Arms Control and The Chemical Weapons Convention

Nova et Vetera Iuris Gentium

Martinus Nijhoff Publishers, Dordrecht, 2001, 202 pp., € 72.00

This volume is the result of a seminar held July 2, 1997 at Utrecht University where a learned audience of academics, diplomats and (int.) civil servants, all experts in either general international or in the work of the Organization for the prohibition of Chemical Weapons (OPCW), debated two subjects.

The morning session dealt with the obligations inter se of the signatories of the Chemical Weapons Convention, an international law topic which has specific relevance for arms control treaties given the security interests of State Parties to these treaties in maintaining a minimal/minimum status quo between all signatories. In this respect the case of the Chemical Weapons Convention was illustrated because in May 1997 at the first conference 165 states had ratified it, Russia being among those who had only signed it while being one of the two principal possessors of chemical weapons. When this book was published in September 2000, though, 174 states had signed. Certainly this issue is highly relevant for other arms control treaties like the Comprehensive Test Ban Treaty (CTBT) which in September of 2000 was not yet in force.

The afternoon session was devoted to the institutional aspects of the OPCW and its supervisory functions. Since this newly established international organization is the first completely devoted to supervision of arms control commitments the topic is of major relevance for the law of international organizations in general.

Following a quick introduction of the contributors to the volume Utrecht University's Erik P.J. Myjer - in the first chapter - introduces the core subject "The Law of Arms Control, Military Security and the Issues". He begins with the question if indeed there is such a separate area within international law that deserves to be distinguished as arms control law and how it relates to other areas with regards to arms, force and its application, namely the 
law of collective security and humanitarian law of armed conflict. Naturally in this book the starting point is the position that arms control law as a separate area of international law indeed exists and that the Chemical Weapons convention is a treaty belonging to it. When the setting is evident the author explains the concept of military security and the ban of the use of force, the law of military security and the relationship between the law of arms control, the law of collective security and the humanitarian law of armed conflict.

The second chapter by Jan Klabbers from Finland is called "Strange Bedfellows: The Interim Obligation and the 1993 Chemical Weapons Convention". He outlines the Chemical Weapons Convention (CWC) and discusses Art. 18 of the Vienna Convention of the Law of Treaties, identifies the object and purpose of the CWC and describes the conditions under which acts can be said to actually defeat the object and purpose of a treaty pending ratification or entry into force and adds a brief word on the principle of estoppel.

In their comments the Dutch E.W. Vierdag, Thilo Marauhn from Heidelberg and Jerzy Sztucki from Uppsala call the speech 'lucid and learned' and list their agreements and disagreements offering a broad scope of interesting though so each reader can then draw his own conclusions based on a list of pros and cons. This is followed by a short summary of the most important issues by Tim Sneek from The Hague.

The third chapter about "The Organization for a Prohibition of Chemical Weapons: Closer Towards an International Arms Control Organization? A Quantum Leap in the Institutional Law of Arms Control" is by Eric Myjer again. After showing the historical development of the control of chemical weapons he describes first the CWC and then the OPCW in general. Extensively he deals with the supervisory functions of both OPCW and CWC and in his concluding remarks stresses the relevance of initial organizational practice. Here critical comments are by Dieter Fleck from Bonn, Johan Rautenbach from The Hague and Thilo Marauhn; again Tim Sneek provides the summary of discussion.

At the end of the book we find the text of the Convention on the Prohibition of the Development, Production, Stockpiling and Use of Chemical Weapons and on their Destruction, in the version of August 8, 1994, which is a very helpful addition as one can simply turn back and check the relevant articles while reading the essays and comments.

There is an occasional problem with the English language, some parts of the work do not seem fluent or idiomatic, but this does not distract much from the well-presented content and bright yet controversial thoughts. The volume is an interesting and challenging read, and a valuable source of information for diplomats, academics and practitioners. Yet one wonders whether Dr. Fleck's statement that it would take approximately 10 years to make a change in this area of international law was realistic when written as it appears much too positive in 2002. Still, this is a comprehensive analysis highlighting various aspects that are of major importance in evaluating the contribution of this particular convention to the institutional law of arms control.

Dagmar Reimmann, Tong Norton, England 


\section{Markus Kotzur}

\section{Theorieelemente des internationalen Menschenrechtschutzes}

Das Beispiel der Präambel des Internationalen Paktes über bürgerliche und politische Rechte

Duncker \& Humblot Verlag, Berlin, 2001, 371 S., $€ 74,00$

Eine der bemerkenswertesten Entwicklungen der Nachkriegsepoche ist die Entwicklung einer globalen Menschenrechtskultur. Diese Menschenrechtskultur ist dabei nicht nur ein ethisches Postulat oder gar nur ein rhetorisches Reservoir zur Verschönerung von Sonntagsreden, sondern eine in verschiedenen Formen mit den Mitteln des positiven Rechts gehärtete Realität geworden - brüchig, unvollkommen, von Machtinteressen beschränkt, zerstörbar zweifellos, aber sichtbar genug, dass ein Prozess menschrechtsorientierter Konstitutionalisierung nicht nur in nationalen Rechtsordnungen, sondern auch auf internationaler Ebene festzuhalten ist. Eine juristische Frage, die sich im Hinblick auf diese Entwicklung stellt, ist, ob überhaupt, wenn ja, an welchem systematischen Ort der Grundrechtsdogmatik Menschenrechtstheorien eine Rolle spielen und welche der verschiedenen (naturrechtlichen, kontraktualistischen, prozeduralen, kulturrelativistischen, utilitaristischen, vernunftrechtlichen, ontologischen, mentalistischen etc.) Grundrechtstheorien vor dem Richtstuhl wissenschaftlicher Reflektion bestehen kann.

Das Buch von Kotzur versucht, einigen dieser Probleme durch das Aufspüren und Erwägen von „Theorieelementen“ im internationalen Menschenrechtsschutz näherzutreten. Der Ausgangspunkt der Arbeit ist eine kulturwissenschaftliche Grundrechtstheorie. Ihre theoretischen Grundideen nebst manchem Argument im Einzelnen gewinnt sie dabei durch Rezeption von Häberles kulturwissenschaftlicher Verfassungstheorie (S. 6 ff.). Ausgangspunkt ist dabei die These, dass einerseits eine zu kasuistische Völkerrechtslehre zu eng konzipiert, eine Theoriebildung also unausweichlich sei (S. 9). Es gebe aber „keine einheitliche, abschließende, ,richtige' Menschenrechtstheorie“" (S. 1). Negatives Gegenmodell der Arbeit ist der Rechtspositivismus, namentlich Kelsen, der an verschiedenen Stellen als Beispiel verengter Rechtslehre angeführt wird (z.B. S. 9 f.), als eine ,ausschließlich dogmatisch arbeitende normative Wissenschaft", die übersehe, dass Recht selbst ein Stück Kultur bilde, dass die Rechtsquellen Produkt außerrechtlicher Entwicklungen seien. Vom kulturwissenschaftlichen Ansatz ergebe sich dagegen die entscheidende Erkenntnis: Die rechtliche Urteilsfindung sei ein „Kulturgespräch“ der im internationalen Rahmen betroffenen Kulturkreise (S. 12). Damit sei die Notwendigkeit einer ,multidisziplinären“ Analyse vorgezeichnet, die Hermeneutik (S. 25 ff.), ,wirklichkeits- und erfahrungswissenschaftliche“ Ansätze (S. 37 ff.), rechts- und kulturvergleichende Studien (S. 47 ff.) sowie die Rezeption von Theorieelementen der nationalen Staats- und Verfassungslehren (S. 56 ff.) verbinde.

Die Studie wendet sich dann im zweiten Teil der Präambel des Internationalen Paktes über bürgerliche und politische Rechte (IPbürgR) zu und bietet Erwägungen zum Begriff der „Präambel“ (S. 60 ff.), zur Form (S. 65 ff.), Sprache (S. 78 ff.) sowie zu weiteren 
spezifischen Funktionen wie Normsetzungen (S. 103 ff.), Interpretationsanleitung (S. 105 ff.) oder Stellungnahme zur Vergangenheit und Zukunft (S. 109 ff.), die Präambeln erfüllten.

Im dritten Teil wird dann unternommen, das „Menschenrechtsprogramm“ der Präambel des IPbürgR zu entwickeln. Dabei wird sich Grundfragen des Menschenrechtsschutzes durch Verträge zugewandt (S. 120 ff.), insbesondere den Fragen der erga omnes-Wirkung der Verträge und der Möglichkeit, Menschenrechtspakte als „Verfassungsverträge“ oder sogar Friedensverträge in einem weiteren Sinne zu verstehen. Sie beschreibt dann die Völkerrechtskonzeption, die in der Präambel enthalten sei (S. 138 ff.), die zu einem anthropozentrischen Völkerrecht führe und erwägt dann den Gehalt der Idee einer verfassten Völkerrechtsgemeinschaft (S. 146 ff.). Das nächste Element ist der Staatsbegriff des IPBürgR, der auf einen menschenrechtsorientierten Verfassungsstaat weise (S. 165 ff.). Eine Erläuterung zur internationalen Öffentlichkeit folgt (S. 180 ff.). Dann werden materiale Menschenrechtsgehalte im Überblick skizziert: Menschenwürde (S. 217 ff.), Freiheit, Gerechtigkeit und Frieden (S. 233) werden dabei berührt. Auch das „Prinzip Verantwortung“ wird als Theorieelement des internationalen Menschenrechtsschutzes eingeordnet (S. 274 ff.). Im Zusammenhang mit letzterem nimmt die Studie vorsichtig zugunsten von Menschen- und Grundpflichten Stellung (S. 307 ff.). Die Debatte um die Mediatisierung staatlicher Souveränität im internationalen Rechtsraum wird dann in Grundzügen reflektiert (S. 313), bevor Überlegungen zur Universalität der Menschenrechte das Buch beschließen (S. 328). Hier wird eine zentrale These erneut im Anschluss an Häberle entfaltet: Der Gegensatz von Universalität und Relativität von Menschenrechten sei zugunsten von Universalität und Partikularität aufzulösen: Die Universalität der Menschenrechte sei zwar zu verteidigen, aber eine „Fiktion“, da die Menschenrechte nur in partikularen Rechtskulturen Gestalt gewönnen (S. 12, 328 ff.). Die Universalität der Menschenrechte sei ein Prozess, kein Zustand.

Die Präambel des IPbürgR dient bei diesen Ausführungen im wesentlichen als Anlass der Entwicklung der Probleme, konkrete normative Konsequenzen für das Verständnis der Präambel oder des Paktes werden dagegen kaum gezogen.

Ein Problem der Arbeit ist die Vielfalt der angesprochenen „Theorieelemente“ und ihr Verhältnis zueinander. Die Studie bezieht sich zustimmend z.B. auf Rawls' Gerechtigkeitstheorie (S. 122), Diskurstheorien der Legitimation (S. 163), klassische und moderne Gesellschaftsvertragstheorien (S. 168, 321: Weltgesellschaftsvertrag), Systemtheorie (S. 211), Jonas' (ontologische) Verantwortungsethik (S. 274), den Kommunitarismus (S. 276) oder auf Kants kategorischen Imperativ (S. 256, 310). Diese Theorien werden durchweg nicht in Voraussetzungen und Konsequenzen entwickelt und dann einer standpunktbestimmenden Kritik unterzogen, sondern schlagwortartig angeführt. So bleibt ungeklärt, ob und wie ihre sich oft gegenseitig ausschließenden Grundvoraussetzungen miteinander in Einklang bringen lassen.

Ein weiteres Problem betrifft die Fundierung der Grundinhalte der Menschenrechte. Sie seien ,angeboren, unabdingbar und unveräußerlich“ (S. 128), ergäben sich aus der ,univer- 
sellen Bedürfnisstruktur" der Menschen, die sie als Staatsaufgaben konstituierten (S. 173), und bauten auf drei Grundprinzipien des Naturrechts auf, die auch für die „Menschenrechtskonzeption des IPBürgR wegleitend“ seien, nämlich, dass sie in der menschlichen Natur begründet lägen, ,universell und unveränderlich, unabhängig vom sozialen, politischen oder kulturellen Kontext" und Individual- und nicht Gruppenrechte seien (S. 223). Die Menschenwürde sei (ein Zitat Dürigs, das als „maßgeblich“ hervorgehoben wird) eine „Seinsgegebenheit, die unabhängig von Zeit und Raum ,ist' und rechtlich verwirklicht werden , soll'“ (S. 229) und letztlich auf das kulturübergreifende Allgemeingut des „Allgemein-Menschliche(n)“, die Bedürfnisstruktur, den Achtungsanspruch, die Verletzlichkeit der Menschen zurückführbar (S. 221). Die Frage ist: Wie sind diese (metaphysik- und naturrechtsnahen) Formulierungen mit dem betonten Wertepluralismus (S. 235) vereinbar? Wie sind sie mit dem gleichberechtigten Kulturgespräch vereinbar, das global die Universalität der Menschenrechte erst bilden soll? Hier werden Maßstäbe vorausgesetzt, die das Kulturgespräch anleiten und die kulturellen Sphären (wenn man solche überhaupt im Hinblick auf interne Heterogenität klar abgrenzen kann, zweifelnd dazu der Autor selbst S. 52 f.) transzendieren. Ist es dann nicht überzeugender sich zu einer universalistischen Menschenrechtstheorie zu bekennen, statt ein konfliktaufhebendes Jenseits von Universalismus und Relativismus zu behaupten? Ist damit nicht auch im Kernbereich der Inhalte der Menschenrechte der Schritt zur Formulierung einer ,richtigen Menschenrechtstheorie“ bereits vollzogen? Sollte man nicht auch das offen aussprechen und dann versuchen zu verteidigen? Schließlich: Was heißt „Fiktion“ der Universalität eines Rechts und partikulare Realisierung genau, die dieses Recht in die Realität übertragen soll? Wann ist die Grenze von der partikularen Realisierung eines Rechts zur Verletzung überschritten? Nach welchen Maßstäben bestimmt sich dies? Welche Bedeutung hat dies für die konkrete internationale Menschenrechtsdogmatik?

Die Arbeit bietet so insgesamt einen Überblick über verschiedene wichtige Fragen des internationalen Menschenrechtsschutzes, der über kulturwissenschaftliche Perspektiven auf diesen Problemkreis engagiert informiert.

Matthias Mahlmann, Berlin 


\section{Erwin Müller / Patricia Schneider / Kristina Thony (Hrsg.)}

\section{Menschenrechtsschutz}

Politische Maßnahmen, zivilgesellschaftliche Strategien, humanitäre Interventionen

Nomos-Verlagsgesellschaft, Baden-Baden, 2002, 386 S., € 42,00

„Human Rights are not theoretical, they are life and death issues.“

Mary Robinson

Der Sammelband ist die dritte Publikation der im Rahmen der Schriftenreihe „Demokratie, Sicherheit, Frieden“ erscheinenden Unterreihe „Frieden durch Recht“ des Instituts für Friedensforschung und Sicherheitspolitik der Universität Hamburg.

„Eine Norm, deren Einhaltung im Belieben des Normadressaten steht, verkommt letztlich (...) zur Farce. Aus diesem Grund gewinnt die Frage nach den Mechanismen und Instrumenten des Schutzes der Menschenrechte bzw. ihrer gewaltlosen Durchsetzung fundamentale Bedeutung“ (S. 21). Antworten auf diese Frage versuchen die Beiträge dieser Anthologie zu geben, die Laien eine gute Einführung in die Thematik, Experten die Chance zur Erweiterung ihres Wissens bieten.

Unterteilt in zwei Hauptabschnitte und einen Epilog befasst sich die Sammlung im ersten Teil mit politisch-diplomatischen Mitteln, im zweiten Teil mit dem militärischen Mittel der humanitären Intervention. Nach einer zweifachen Einleitung, die in Deutsch und Englisch abgefasst ist, widmet sich Teil 1 den Instrumenten des Menschenrechtsschutzes insbesondere im Rahmen der Arbeit internationaler Organisationen, aber auch der nicht-staatlichen Menschenrechtsorganisationen der Zivilgesellschaften. Die Rede Kofi Annans vor der Konferenz in Den Haag am 18. Mai 1999 aus Anlass der 100-Jahr-Feier der Haager Friedenskonferenz leitet den ersten Teil ein. Im Mittelpunkt steht das Ideal einer Herrschaft des Rechts; das Nichterreichen dieses Ideals und die Tatenlosigkeit seiner Organisation kritisiert der UNO-Generalsekretär.

Anja Jetschkes Beitrag beschäftigt sich mit der Frage, wie die in internationalen Konventionen verankerten Menschenrechte durchgesetzt werden können, aus einer politikwissenschaftlichen Perspektive, d.h. aus der der internationalen Beziehungen, um die Möglichkeiten und Grenzen der Durchsetzung von Menschenrechtsnormen zu erörtern. Sie zeigt auf, wie Netzwerke von transnationalen Menschenrechtsorganisationen zur Durchsetzung von international verankerten Menschenrechtsnormen beitragen. Zunächst macht sie eine Bestandsaufnahme der weltweiten Durchsetzung von Menschenrechten, danach werden die verschiedenen Instrumente zu ihrer Durchsetzung diskutiert. Die Autorin befasst sich mit der Rolle und Funktion von transnationalen Netzwerken von NGOs und analysiert abschließend die politische Dynamik, die sich aufgrund der Interventionen ergeben kann, sowie mögliche Abweichungen vom Ideal des Modells.

Das Thema von Nils Geißler ist „Einfluß und Rolle der Nichtregierungsorganisationen beim Schutz der Menschenrechte“, die oft die letzte Chance für die Opfer sind. „The Challenge of Implementing Human Rights Law: An Overview of the Role Played by the 
United Nations System“ ist der Beitrag von Mark Freeman, der die Kompetenzen des Menschenrechtsschutzes aufzeigt und ihre Defizite analysiert.

Die VN-Menschenrechtskommission, eine der prominentesten Einrichtungen, handelt der Aufsatz von Wolfgang Heinz ab. Dieser Beitrag beginnt mit einem Überblick zu den VNOrganen und -Organisationen, die sich mit Menschenrechten befassen, und stellt die wichtigsten Aspekte der VN-Menschenrechtskommission dar. Thema sind die allgemeinen Aspekte einer Reformdiskussion sowie die konkrete Debatte der letzten Jahre. Trotz einiger Reformen bedarf es der Zuweisung weiterer Kompetenzen, um der Aufgabenstellung gerecht werden zu können.

„Möglichkeiten und Grenzen der menschlichen Dimension der KSZE/OSZE - von der Schlussakte von Helsinki (1975) zum Istanbuler Gipfel (1999)“: Randolf Oberschmidt geht der Wirksamkeit der wichtigsten normativen Grundlagen der menschlichen Dimension nach der Klärung dieses Begriffs - sowie ihrer Instrumente und Institutionen nach und bietet einen interessanten Ausblick auf ihren zukünftigen Stellenwert im Rahmen der OSZE.

Christa Stolle schildert die Verletzungen der Menschenrechte von Frauen in „Menschenund Bürgerrechte als Frauenrechte“. „Gewalt gegen Frauen ist ein gemeinsames Merkmal aller patriarchalischen Gesellschaften“ (S. 155), kennt keine Grenzen und ist universal. Hier gibt es detaillierte Einblicke in das Leben der Frauen in Afghanistan, den langen Marsch durch die Institutionen für Frauenrechte, das internationale Frauenjahr von 1972, die UNO-Konferenzen von 1975/1980/1985, Erfolge auf diesem Gebiet und die Arbeit des Vereins „Terre des Femmes“ in Tübingen.

Der letzte Beitrag in diesem Teil stammt von Kinga Gál mit dem Aufsatz „The Implementation of Minority Rights“. Der Aufsatz zeigt die Menschenrechte als individuelle Rechte sowie Minderheitenrechte als Kollektivrechte auf nationaler, regionaler und globaler Ebene.

Teil 2 des Sammelbandes befasst sich mit der „Fortsetzung der Politik mit anderen Mitteln“, nämlich mit der Dimension des Schutzes der Menschenrechte durch Gewalt in Form der sogenannten humanitären Intervention. Birthe Ankenbrand referiert die Kriterien einer humanitären Intervention - ein Problem, das durch den NATO-Militäreinsatz im KosovoKrieg eine neue Diskussion entfacht hat -, die für die Einsätze aufgestellt werden, um willkürliches Vorgehen unter einem humanitären Vorwand ausschließen zu können.

Ausgangspunkt Rolf Schüßlers ist der historische Bezug. Er vergleicht die mittelalterliche Doktrin des bellum iustum mit dem Konzept der humanitären Intervention und befasst sich mit ihrer moralischen Legitimation. „Die Frage, ob nicht nur interveniert werden darf, sondern interveniert werden muss, um Rechtsverletzungen höchsten Ausmaßes zu beenden, benennt eines der ethischen Grundprobleme der humanitären Intervention“ (S. 210).

Der Aufsatz Reinhard Marx’ „Humanitäre Intervention aus der Sicht der Menschenrechtsbewegung " macht deutlich, dass jede beziehbare Position ambivalent ist: Die Gewaltfreiheit als Ideal steht der Ethik des Hilfsangebots für die Opfer solcher Menschenrechtsverletzun- 
gen entgegen, dem vielleicht ausschließlich mit Waffengewalt Einhalt geboten werden kann.

Kirsten Sparre bespricht sowohl die historischen wie auch die aktuellen Erfahrungen mit der Rolle der Massenmedien im Rahmen der Berichterstattung über Kriege und bewaffnete Konflikte. Wünschenswert wäre eine kritisch-beobachtende journalistische Ethik, insbesondere in bezug auf die Position von Regierungen.

Jamie Shea, der hier seine persönliche Meinung publiziert und nicht den offiziellen Standpunkt der NATO einnimmt, beschreibt in seinem Bericht über moderne Konflikte anhand des Kosovo-Krieges seine eigenen Erfahrungen in der Öffentlichkeitsarbeit und zeigt mögliche Lehren daraus auf. Nach der Vermeidbarkeit oder Notwendigkeit des KosovoKrieges der NATO fragt Matthias Z. Karádi. Der Verfasser listet die Argumente dafür und dagegen auf und legt besonderes Augenmerk auf die schwierige Situation der politischen Entscheidungsträger. Nachdenklich macht der Text von Elke Steven, der sich damit befasst, wie deutsche Gerichte mit Gegnern des Kosovo-Krieges umgegangen sind.

Mark Mangers Thema „Humanitäre Intervention - auch gegen die eigenen politischen Interessen?" ist gerade jetzt im Herbst 2002 wieder ein sehr aktueller Titel. Er spürt dem Spannungsverhältnis zwischen nationalen Interessen und übergeordneten humanitären Normen nach.

Im Epilog - der leider nicht wie die Einleitung sinnvollerweise ins Englische übersetzt wurde - zieht Patricia Schneider eine Bilanz des heutigen Standes der Normierung der Menschenrechte, ihrer Achtung und ihres Schutzes. Sie beschreibt den langen Weg zum Ziel, erinnert an die wichtigsten Meilensteine und analysiert das Maß der tatsächlichen Verwirklichung. Eine bessere internationale Gerichtsbarkeit auf diesem Gebiet mahnt die Autorin an.

Manche Beiträge enthalten Schaubilder und Tafeln, fast alle ein eigenes Literaturverzeichnis. Das Buch ist solide und lehrreich, in beiden Sprachen verständlich geschrieben, meist gut strukturiert und kenntnisreich; es deckt viele spannende Teilbereiche ab und enthält auch eine ausführliche Gesamtbibliographie.

"This is the core challenge of the Security Council and the United Nations as a whole in the next century: to unite behind the principle that massive and systematic violations of human rights conducted against en entire people cannot be allowed to stand. (...) On the eve of the new millennium, it is this United Nations we seek - responsive to a dynamic and changing world, respectful of the sovereignty of States, and resilient in its determination to advance the rights and freedoms of the peoples in the world."

Kofi Annan

Dagmar Reimmann, Tong Norton, England 


\section{Margarita González}

\section{Der völkerrechtliche Status des Panamakanals nach Ablauf der Übergangszeit im Jahr 1999}

Schriften zum Staats- und Völkerrecht, Band 84

Peter Lang Verlag, Frankfurt/Main, 2000, 212 S., € 35,30

Im Jahre 1977 schlossen die Vereinigten Staaten und Panama einen Vertrag, der die schrittweise Übergabe der zweitwichtigsten künstlichen Wasserstraße der Welt sowie der in der seit 1903 von den USA autonom verwalteten Kanalzone befindlichen Einrichtungen an den Isthmusstaat vorsah. Seit dem Sylvestertag 1999 genießt Panama die volle Souveränität über den Kanal und die angrenzenden Gebiete; viele der Anlagen auf den früheren Militärbasen der US-Streitkräfte konnten zwischenzeitlich zivilen Zwecken zugeführt werden.

Das Vertragswerk von 1977 besteht aus zwei Teilen: dem eigentlichen Kanalvertrag, der im wesentlichen die Fristen und Modi der Übertragung der Kanalverwaltung definiert und dessen Geltungsdauer mit dem Ablauf der Übergangszeit endete, sowie dem sog. Neutralitätsvertrag, der Bestimmungen über den Betrieb und die Verteidigung des Kanals ab dem Jahr 2000 enthält. Die Reichweite der darin den USA gewährten Befugnisse ist umstritten. Vor dem Hintergrund der jahrzehntelangen Beschränkung der panamaischen Souveränität durch die seit dem ersten Kanalvertrag von 1903 den USA gewährten Sonderrechte geht die Autorin der Frage nach, ob die Bestimmungen des Neutralitätsvertrages einschließlich der während des Ratifizierungsverfahrens vom US-Kongress hinzugefügten Vertragszusätze Washington ein Recht auf militärische Interventionen in Panama einräumen. Gleichzeitig möchte sie den im Vertrag verwendeten Begriff der „Neutralität“ einer kritischen Betrachtung unterziehen sowie den ,im Völkerrecht umstrittenen Problemkreis der garantievertraglichen Interventionsrechte und ihrer Vereinbarkeit mit dem völkerrechtlichen Gewaltund Interventionsverbot" (S. 21) behandeln.

Teil A enthält eine knappe Skizze der Geschichte des Kanals und der Kanalverträge bis zum derzeit geltenden Vertrag. Teil B präsentiert eine Analyse der Regelungsgegenstände der Carter-Torrijos-Verträge von 1977, wobei der (Ende 1999 ausgelaufene) Kanalvertrag nur kurz behandelt wird. Im Hinblick auf den Neutralitätsvertrag macht die Verfasserin deutlich, dass die in Art. VI verfügte und in einem der vielen Vertragszusätze (Understanding 1) präzisierte Privilegierung von US-Kriegsschiffen beim Kanaltransit gegen den eigentlichen Kern des Neutralitätsprinzips verstößt. Als mit der vollen Souveränität des Kanalstaats unvereinbar und konfliktträchtig beurteilt González die ebenfalls in Understanding 1 näher erläuterte Vorschrift zur Anpassung der Transitgebühren, in der die Interessen der USA als Handelsmacht durchscheinen.

In Kapitel $\mathrm{C}$ werden grundsätzliche Fragen in Bezug auf die Anwendung der Wiener Vertragsrechtskonvention bei der Auslegung der Carter-Torrijos-Verträge geklärt. Nach generellen Ausführungen zu den völkerrechtlichen Interpretationsprinzipien charakterisiert die Autorin die Kanalverträge von 1977 in Anlehnung an einen vom deutschen Bundesverfassungsgericht im Zusammenhang mit den sog. „Ostverträgen“ geprägten Begriff als „hoch- 
politische Verträge“. Sie begründet dies mit der außerordentlichen politischen Bedeutung, die den Verträgen auf beiden Seiten beigemessen wurde. Während Panama damit die seit Jahrzehnten herbeigesehnte Verwirklichung der ungeschmälerten Souveränität über sein Territorium verband, waren die USA bestrebt, ihre wirtschaftlichen und geostrategischen Interessen auch unter dem neuen Statut so weit wie möglich zu wahren. Um trotz z.T. nicht $\mathrm{zu}$ vereinbarender Positionen zu einem Vertragsabschluss zu kommen, waren damals in einige Paragraphen bewusst unklare Formulierungen aufgenommen worden, damit jede Seite die gewünschte Interpretation herauslesen konnte. Gerade in einem solchen Fall sieht die Autorin die Notwendigkeit einer restriktiven Auslegung der Verträge gegeben. Damit präformiert sie freilich in starkem Maße das Resultat ihrer weiteren Untersuchungen.

Die Untersuchung des Neutralitätsvertrages in Kapitel D führt zu dem Schluss, dass die USA als Garant des Neutralitätsstatus des Kanals zwar die Pflicht haben, im Falle einer Gefährdung dieses Zustands tätig zu werden, dabei aber keine militärischen Mittel einsetzen dürfen. Nach Auffassung der Autorin ist ein einseitiges militärisches Interventionsrecht auch mit Artikel 5 des Vertrages unvereinbar, der besagt, dass ab dem Jahr 2000 nur noch Panama berechtigt ist, innerhalb seines Staatsgebiets militärisch präsent zu sein. Dass starke politische Gruppen in Washington eine solche Interpretation nicht teilen, ist allgemein bekannt. Allerdings sind es in erster Linie die diversen Vorbehalte, welche Angehörige des US-Kongresses während des Ratifikationsverfahrens durchzusetzen vermochten, an denen die großen Interessengegensätze zwischen den Vertragspartnern deutlich werden. Ihre rechtliche Tragweite erhalten diese Zusätze durch die Tatsache, dass sie von panamaischer Seite angenommen wurden; damit bilden sie primäre Auslegungsmittel bei der Interpretation der Verträge. Die Analyse der Vertragszusätze (S. 81-119) stellt den interessantesten Teil des Buches dar.

In Amendment 1 macht González zwei Modifikationen in Bezug auf den Neutralitätsvertrag aus: Erstens wird die Pflicht der USA zur Verteidigung des Neutralitätsstatus des Kanals zu einem Recht erweitert, zweitens wird Washington die Möglichkeit eingeräumt, autonom d.h. ohne Absprache mit Panama - Maßnahmen zum Schutz der Neutralität des Kanals zu ergreifen. Die Autorin legt zwar dar, dass die USA diesen Zusatz als Bestätigung bzw. Präzisierung eines einseitigen militärischen Interventionsrechts betrachten, sie kommt bei ihrer völkerrechtlichen Interpretation des Amendments jedoch zum gegenteiligen Ergebnis. Ohne Verklausulierung kommt die sog. Condition DeConcini daher, in der ein unilaterales militärisches Interventionsrecht der USA offen deklariert wird. Panama hat zwar anerkannt, dass dieser Zusatz in Bezug zum Neutralitätsvertrag steht, dessen Inhalt aber nicht akzeptiert. Panamas Proteste gegen den DeConcini-Zusatz resultierten in der sog. Reservation Church, die ausdrücklich das völkerrechtliche Interventionsverbot hervorhebt und das Interventionsrecht der USA allein auf den Schutz des neutralen Status des Kanals bezieht. Während Panama in diesem Zusatz (offiziell) eine wirksame Blockierung der Bestimmungen der Condition DeConcini sieht, legt die Autorin überzeugend dar, dass es sich hierbei eher um ein diplomatisches Instrument handelt, das die Bestimmungen des von Senator DeConcini eingebrachten Zusatzes nicht wirklich beschränkt. 
Die Untersuchung erstreckt sich auf zwei weitere Zusätze, die den behandelten Fragenkomplex betreffen. Understanding 2 wiederholt und bekräftigt im Wesentlichen nur zentrale Bestimmungen von Amendment 1 sowie der Condition DeConcini. Die sog. Condition Nunn besagt, dass Panama und die USA auch nach 1999 Abkommen über die Stationierung von US-Militär auf panamaischem Boden schließen können. Nicht nachvollziehbar ist die These der Autorin, dass diese Condition eine Modifikation des Neutralitätsvertrags darstelle, weil sie im Widerspruch zu dessen Artikel 5 stehe, der ab dem Jahr 2000 die alleinige Militärpräsenz Panamas auf seinem Staatsgebiet postuliert: Condition Nunn betont lediglich die Möglichkeit solcher Verhandlungen, und bei Einigkeit beider Seiten können bestehende vertragliche Bestimmungen leicht revidiert werden. Hinsichtlich der drei von panamaischer Seite in die Ratifikationsurkunde integrierten Erklärungen gelangt González zu dem Schluss, dass sie wenig Gewicht bei der Auslegung der Verträge besitzen, weil sie nicht viel mehr als die Betonung allgemeiner völkerrechtlicher Grundsätze (Interventionsverbot, nationale Selbstbestimmung) enthalten. Als Ergebnis ihrer Analyse des Neutralitätsvertrages und der erwähnten Zusätze konstatiert die Verfasserin einen unverkennbaren Dissens über Ausmaß und Wesen der den USA zustehenden Interventionsrechte. Aufgrund der Befolgung des Grundsatzes der restriktiven Auslegung liegen ihre Schlussfolgerungen zwangsläufig nahe bei der panamaischen Interpretation des Vertragswerks. Ihrer Argumentation zufolge würde ein militärisches Interventionsrecht der USA nur dann vorliegen, wenn Panama einer solch gravierenden Einschränkung seiner Souveränität ausdrücklich zugestimmt hätte. Wenn man jedoch einer weiten Auslegung folge - so die anschließenden Überlegungen der Verfasserin - und vom Tatbestand eines den USA eingeräumten militärischen Interventionsrechts ausgeht, wären die Verträge nichtig, weil ihre Bestimmungen sowohl gegen das völkerrechtliche Gewaltverbot als auch gegen das Interventionsverbot verstießen.

Kapitel E geht der Frage nach, ob die Gründe, die die Administration Bush Ende 1989 zur Rechtfertigung ihrer militärischen Intervention gegen den panamaischen Machthaber Noriega genannt hat, einer völkerrechtlichen Prüfung standhalten. Die USA hatten die Militäraktion, in deren Verlauf rd. 300 Personen getötet und - aufgrund der Zerstörung von Wohngebieten durch Bomben - über 10.000 Menschen obdachlos wurden, u.a. mit der Notwendigkeit, ihre Verpflichtungen aus den Carter-Torrijos-Verträgen zu erfüllen, begründet. Wenig überraschend gelangt González zu dem Ergebnis, dass die Anwendung militärischer Gewalt in Panama durch die USA nicht gerechtfertigt war und die Aktion daher als völkerrechtswidrig einzustufen ist.

Gerade im Lichte der Panama-Invasion von 1989 wird der hochgradig akademische Charakter der gesamten Abhandlung deutlich. Zwischen Realpolitik und völkerrechtlichen Prinzipien besteht im vorliegenden Fall eine kaum zu überbrückende Kluft, wobei die konkreten Interessen und Ziele der Supermacht USA als zentrale Determinante fungieren. Die zahlreichen Zusätze zu den Kanalverträgen dienten doch allein der Selbstvergewisserung und Bekräftigung des in Anspruch genommenen militärischen Interventionsrechts. Ohne die mehr oder weniger verklausuliert verbriefte Option, die nationalen Sicherheits- 
interessen auch unter dem neuen Kanalstatut jederzeit unilateral durchsetzen zu können, wären die Carter-Torrijos-Verträge niemals ratifiziert worden. Trotz der großen Zugeständnisse an die konservativen Senatoren waren die Verträge damals nur mit einer Stimme über dem erforderlichen Mehr angenommen worden.

In gewisser Weise präsentiert González in ihrer Monographie die völkerrechtliche Feinanalyse eines Vertragswerks, dessen Relevanz nicht von rechtlichen, sondern weit überwiegend von politischen Faktoren bestimmt wird. Durch die markante Veränderung des in konservativen US-Kreisen perzipierten Bedrohungsszenarios nach dem Ende des Kalten Krieges ist freilich die Chance gewachsen, dass der Dissens bei der Auslegung der Kanalverträge in Zukunft politisch kaum Konfliktstoff schaffen wird.

Karl-Dieter Hoffmann, Eichstätt

Domingo García Belaunde / Francisco Fernández Segado (coordinadores)

\section{La jurisdicción constitucional en Iberoamérica}

Dykinson S.L., Madrid / Ediciones Jurídicas, Lima / Editorial Jurídica E. Esteva, Uruguay / Editorial Jurídica Venezolana, Caracas, 1997, 963 S., € 80,91

Das monumentale Werk behandelt mehr als sein Titel verspricht. Während die Titel vieler heute veröffentlichter anderer Bücher nicht ganz das halten, was der Leser nach dem Titel erwartet, ist der Titel des Werkes „La jurisdicción constitucional en Iberoamérica“ eher ein understatement als eine Übertreibung; denn mehr als einer der Beiträge in diesem umfangreichen Werk greift über den iberoamerikanischen Rechtskreis hinaus. Dies gilt für die einführende Darstellung über die historische Entwicklung und die Modelle der Kontrolle der Verfassungsmäßigkeit aus der Feder von Francisco Fernández Segado (S. 45 ff.) ebenso wie für den Beitrag von Louis Favoreu über Verfassungsgeschichte allgemein (S. $100 \mathrm{ff}$.) und die fast monographische Darstellung der Verfassungsbeschwerde im System der Verfassungsgerichtsbarkeit in der Bundesrepublik Deutschland von Peter Häberle (S. 231 ff.). Der ganz überwiegende Teil der insgesamt 25 Beiträge in diesem Sammelwerk befasst sich dagegen mit der Verfassungsgerichtsbarkeit in Iberoamerika (zu diesem Thema vgl. auch den instruktiven Besprechungsaufsatz von Jürgen Samtleben zu dem Buch von Norbert Lösing, Die Verfassungsgerichtsbarkeit in Lateinamerika, VRÜ 35 (2002), S. 120 ff.). Die Entscheidung von García Belaunde und Fernández Segado, das Werk weder „Justicia constitucional“ noch „Derecho procesal constitucional“ zu nennen oder den von Kelsen gebrauchten Ausdruck, ,judicial review“ zu verwenden (vgl. dazu S. 8), war richtig. Dem Gegenstand des Handbuchs entspricht es auch, dass die Beiträge in spanischer Sprache verfasst sind (die erwähnten Artikel von Favoreu und Häberle sind Übersetzungen); 
lediglich die Beiträge der portugiesischsprachigen Autoren sind in ihrer Muttersprache geschrieben.

Der Inhalt des Werkes lässt sich (ohne dass dies ausdrücklich in der Gliederung entsprechend vermerkt ist) in einen allgemeinen und in einen besonderen Teil untergliedern. Zum allgemeinen Teil gehören die bereits erwähnten Beiträge über die historische Entwicklung, über die Verfassungsgerichte allgemein und über die Verfassungsbeschwerde in Deutschland, aber auch die Beiträge von Allan R. Brewer-Carías über die Verfassungsgerichtsbarkeit in Lateinamerika (S. 121 ff.) und von Héctor Fix-Zamudio über das interamerikanische System des Menschenrechtsschutzes (S. $167 \mathrm{ff}$.). Im besonderen Teil folgt sodann das, was man als Länderberichte bezeichnen kann: Die Verfassungsgerichtsbarkeit in Argentinien (Juan Carlos Hitters, S. 287 ff.); zusätzlich: Prozessuale Instrumente des Schutzes der Menschenrechte in Argentinien (Néstor Pedro Sagüés, S. 311 ff.); die Verfassungsgerichtsbarkeit in Bolivien (Benjamín Miguel Harb, S. 337 ff.), zusätzlich dazu auch der diesbezügliche Inhalt der Verfassungsreform von 1994 (Francisco Fernández Segado, S. 359 ff.). Brasilien wird in drei Beiträgen behandelt: Die Kontrolle von Gesetzen auf ihre Verfassungsmäßigkeit (José Afonso da Silva, S. 391 ff.); Die Instrumente des prozessualen Schutzes der Menschenrechte (Luiz Pinto Ferreira, S. 413 ff.); Der Verfassungsgerichtsprozess (José Alfredo de Oliveira Baracho, S. 441 ff.). Die Verfassungsgerichtsbarkeit in Kolumbien wird von Eduardo Cifuentes Muñoz dargestellt (S. 473 ff.), die in Costa Rica von Rubén Hernández Valle (S. 505 ff.), die in Chile von Humberto Nogueira Alcalá (S. 539 ff.), die in Ecuador von Hernán Salgado Pesantes (S. 577 ff.), die in El Salvador von Salvador Enrique Anaya Barraza (S. 595 ff.), die in Guatemala von Francisco Fernández Segado und von Domingo García Belaunde (S. 717 ff.), die in Mexiko von Jorge Carpizo, José Ramón Cossío Díaz und Héctor Fix Zamudio (S. 474 ff.), die in Panama von Francisco Rodríguez Robles (S. 809 ff.), die in Peru von Domingo García Belaunde (S. 831 ff.), die in Uruguay von Eduardo G. Esteva Gallicchio (S. 901 ff.), sowie die in Venezuela von Carlos M. Ayala Corao (S. 933 ff.).

Die Verfassungsgerichtsbarkeit in den beiden europäischen Staaten, die sich der iberoamerikanischen Staatenfamilie zugehörig fühlen, sind von Francisco Fernández Segado (Spanien, S. 629 ff.) und von Jorge Manuel Moura Loureiro de Miranda (Portugal, S. 861 ff.) erörtert.

Betrachtet man die mit den genannten Beiträgen bedeckte Landkarte der Verfassungsgerichtsbarkeit Lateinamerikas, so ist das Bild eindrucksvoll. Andererseits gibt es offensichtlich noch einige weiße Flecken - in der Sprache mittelalterlicher Kartographen würde man sagen: hic sunt leones. Nicaragua und Paraguay sind z.B. nicht in dem Werk vertreten. Insgesamt aber zeigt Iberoamerika ein eindrucksvolles Bild der Verbreitung der Verfassungsgerichtsbarkeit in den lateinamerikanischen Staaten. Das hier vorgestellte Werk ist nicht nur für Juristen und Politiker in den genannten Ländern von großem Interesse, sondern auch für den Verfassungsjuristen in Deutschland. Man tritt gewiss keinen Angehörigen dieser (unserer) Zunft zu nahe, wenn man feststellt, dass die Verfassungsgerichtsbarkeit in Iberoamerika hierzulande noch wenig bekannt ist, wenn man von den nicht vielen 
Sachkennern der iberoamerikanischen Verfassungsgerichtsbarkeit absieht und von der allerdings weiter verbreiteten Kenntnis der juicio de amparo nach mexikanischem Recht, einer Art habeas corpus (vgl. dazu S. 779 ff.). Der Wert des von García Belaunde und Fernández. Segado als Koordinatoren herausgegebenen (und von ihnen selbst als Autoren mehrerer Beiträge bereicherten) Werkes kann gar nicht hoch genug veranschlagt werden. Da eine starke, effiziente Verfassungsgerichtsbarkeit ein wesentlicher Pfeiler des Rechtsstaates ist, gibt der Inhalt des Werkes auch Hoffnung für die Intensivierung der Rechtsstaatlichkeit in Übersee.

Ingo von Münch, Hamburg

Klaus Bodemer / Heinrich-W. Krumwiede / Detlef Nolte / Hartmut Sangmeister (Hrsg.)

\section{Lateinamerika-Jahrbuch 2001}

Vervuert Verlag, Frankfurt/Main, 2001, 340 S., € 23,00

Das im Hamburger Institut für Iberoamerikakunde beheimatete Lateinamerika-Jahrbuch muss man hier nicht mehr vorstellen. Das bewährte System, sorgfältige Dokumentation und fundierte Analyse zu kombinieren, zeichnet auch den Jahrgang 2001 aus. Wiederum ist der dokumentarische Teil mit seiner Mischung aus synoptischen Darstellungen, Jahreschronologien (bezogen auf das Jahr 2000) und übersichtlich strukturierten Statistiken zu den Regionalbündnissen und den einzelnen Staaten von großem praktischem Wert für alle, die einen qualifizierten Überblick über die politische, wirtschaftliche und soziale Situation einzelner Staaten oder des Kontinents insgesamt suchen.

Das Augenmerk soll hier im wesentlichen auf den „literarischen“ Teil des Jahrbuches gerichtet werden, der die nüchterne Faktenaufbereitung des dokumentarischen Teils durch die vertiefte Beschäftigung mit bestimmten ausgewählten Themen ergänzt. Er besteht dieses Mal aus vier Abhandlungen, deren Schwerpunkte weniger im rechtlichen als im wirtschaftlichen und politologischen Bereich liegen.

„Die neue Welle regionaler Integration in Lateinamerika aus europäischer Perspektive“ analysiert Peter Nunnenkamp (Institut für Weltwirtschaft, Kiel). Er geht der Frage nach, welche Perspektiven die vier Lateinamerikanischen Regionalbündnisse Mercosur, Andengemeinschaft, CACM (Mittelamerika) und Caricom (karibischer Raum) einerseits für die betreffenden Regionen selbst, andererseits und nicht zuletzt aber auch für die EU-Staaten eröffnen. Sein Befund klingt eher ernüchternd: Die lateinamerikanischen Staaten seien nicht nur durch die o.g. Regionalbündnisse miteinander verbunden, sondern hätten teilweise weitere bilaterale oder unilaterale Abkommen geschlossen, deren Inhalt und Sinn nicht immer mit den Regionalbündnissen kompatibel sei, was zu verworrenen Situationen führen könne. Außerdem gebe es gewaltige Unterschiede zwischen manchen Ländern der 
jeweiligen Gemeinschaften, was den Schluss nahe lege, dass der relative Erfolg mancher Staaten eher auf dortige regionale Besonderheiten als auf die Bündnismitgliedschaft zurückzuführen sei. Der Außenhandel der Gemeinschaften mit Drittländern schließlich habe zwar im Zuge der unilateralen Importliberalisierung in den Gemeinschaften deutlich zugenommen, doch habe dies wegen der ungünstigen Exportergebnisse (fehlende Wettbewerbsfähigkeit) zu einer deutlich negativen Außenhandelsbilanz geführt. Von letzterem wiederum profitiere die EU aber nur begrenzt, da die Importe der Gemeinschaften aus der EU eher gering seien, insbesondere im Vergleich zu Nordamerika; lediglich im Bereich des Mercosur habe die EU ein leichtes Übergewicht. Zwar könnten sich eigentlich neue Chancen für Europa ergeben, da es einerseits lateinamerikanische Bestrebungen gebe, die Abhängigkeit von Nordamerika zu lockern, und andererseits Lateinamerika aus europäischer Sicht eine der wichtigsten Wachstumsregionen sei. Es erscheine jedoch fraglich, ob die EU in der Lage sei, sich etwa im Agrarbereich für Importe zu öffnen; durch ihre ,,agrarpolitische Starre" gefährde sie mögliche eigene Exportchancen. Im Mercosur habe die EU zudem bereits an Glaubwürdigkeit verloren, da man ihre Vorgehensweise in den bisherigen Verhandlungen als einen Ausdruck relativen Desinteresses und als Hinhaltetaktik empfinde.

Während es hier um die Bedeutung transnationaler Bündnisse für den Wirtschaftsstandort Lateinamerika ging, lotet Christian von Haldenwang (Deutsches Institut für Entwicklungspolitik, Bonn) gleichsam spiegelbildlich dazu aus, wie sich die Qualität der subnationalen (regionalen und kommunalen) Ebenen auf den Wirtschaftsstandort auswirkt: „Standortpolitik - neue Rollen für Regionen und Kommunen in Lateinamerika“. Auch sein Ausblick ist eher skeptisch, jedoch sieht er hier einen Lösungsansatz. Er geht von der Erkenntnis aus, Lateinamerika habe ein Standortproblem im Hinblick auf die Schaffung von Wirtschaftswachstum und dessen breitenwirksamen Einsatz zur Überwindung von Armut, und knüpft daran den Vorschlag, diese Probleme (auch) durch politische und administrative Reformen auf regionaler und lokaler Ebene anzugehen. Auch in Europa habe sich die Erkenntnis durchgesetzt, dass Dezentralisierung in Verbindung mit dem Aufbau „weicher“ Institutionen (ohne „harte“ exekutivische Kompetenzen), die für eine bessere Abstimmung zwischen öffentlichen und privaten Akteuren sorgen sollen, im Sinne eines „Neuen Steuerungsmodells“ sowohl zu Effizienzsteigerungen als auch zu Qualitätsverbesserungen führen können. Die Anwendung dieser Grundsätze in Lateinamerika sei allerdings schwierig. Auch wenn die meisten lateinamerikanischen Staaten mittlerweile durch Verfassungsänderungen die formalen Voraussetzungen für eine wichtigere Rolle von Regionen und Kommunen geschaffen hätten, blieben in der Praxis doch erhebliche Probleme: Es dominierten die traditionellen Ministerialbürokratien mit nachgeordneten Sektionen, während es bei den subnationalen Gebietskörperschaften an technischer und personeller Kompetenz fehle; auch die Organisationen der verfassten Wirtschaft seien oft zu schwach, um als kompetente Partner der öffentlichen Hand wirken zu können. Auch wenn auf lokaler bzw. regionaler Ebene teilweise vielversprechende Ansätze erkennbar seien, handele es sich dabei nicht selten um „Inseln im Meer bürokratischer Insuffizienz“, die eher auf das Wirken von Ein- 
zelpersonen zurückzuführen seien als auf institutionalisierte Anreizfunktionen, denen auf nationalstaatlicher Ebene oft eine Strategie der Reformvermeidung im Wege stehe. Trotz dieser Schwierigkeiten seien Reformen, die zu einer Stärkung der subnationalen Ebenen führten (etwa die Schaffung von Anreizfunktionen für erfolgreiche Standortpolitik durch Beteiligung am Gewerbesteueraufkommen), aber unabdingbar, da Lateinamerika Gefahr laufe, von der weltwirtschaftlichen Entwicklung abgekoppelt zu werden. Dementsprechend sollten die lokalen und regionalen Akteure sowie die gesellschaftspolitischen Organisationen einerseits und die internationale Staatengemeinschaft sowie die entwicklungspolitischen Organisationen andererseits Druck auf die nationalstaatlichen Akteure ausüben, die von den subnationalen Ebenen ausgehenden, vielversprechenden Tendenzen weiter zu fördern.

Über „Wege, Vorschläge, Errungenschaften und Herausforderungen der Frauenbewegung in Lateinamerika“ berichtet Berlindes Astrid Küchemann (Soziologieprofessorin an der Universität Brasilia); dieser Beitrag dürfte unterschiedlich starke Betroffenheit auslösen. Die Autorin rekapituliert Entstehung und Verlauf des Feminismus in Lateinamerika, dessen Themenschwerpunkt bis zum Ende der siebziger Jahre die Diskriminierung der Frau in den Bereichen Arbeit, Lohn und Bildung gewesen sei, während ab den achtziger Jahren mit dem Übergang zu zivilen und demokratischen Regierungen immer mehr die Beziehung zwischen Geschlechtern - etwa (Homo-) Sexualität, Abtreibung, Verhütung - an sich zum Thema geworden sei, was zu einer zunehmenden Militanz der Bewegung und zu Konflikten mit linken Parteien geführt habe, denen frauenspezifische Themen als zu „elitär“ erschienen seien. In den neunziger Jahren sei es zu einem Zerfall der feministischen Bewegung in Gruppen und Sektionen sowie zu einer Tendenz zur Institutionalisierung durch Partizipation an staatlichen Einrichtungen, aber auch zu vielfältigeren Aktivitäten als zuvor gekommen. Außerdem beschreibt die Autorin, mit welchen Instrumenten die „Eroberung des Rechts auf Gleichheit“ auf den Weg gebracht worden sei (1975 „Jahr der Frau“, UN-Antidiskriminierungskonvention von 1979, Gesamtamerikanische Konvention zur Vorbeugung, Ahndung und Beseitigung der Gewalt gegen Frauen von 1994, Programme und Aktionspläne der UN-Konferenzen sowie nationale Förderprogramme in einzelnen lateinamerikanischen Staaten), um dann die Aufmerksamkeit auf die Verbreitung des sog. „GenderAnsatzes“ zu richten: Dabei handelt es sich um eine „Analysekategorie für die Erklärung der Unterdrückung der Frau“, welcher ,die soziokulturellen Rahmenbedingungen bestehender Geschlechterdisparität umfassend berücksichtigt“. Die Autorin stellt in diesem Zusammenhang fest, der „Gender-Ansatz“ habe sich immer stärker in Lateinamerika verbreitet, was auch daran liege, dass die Bereitstellung mancher Mittel der Entwicklungszusammenarbeit von der „Gender-Sensibilität“ der Regierungsapparate abhängig gemacht werde. Im Hinblick auf die Zukunftsaussichten der feministischen Bewegung in Lateinamerika konstatiert die Autorin trotz wichtiger Erfolge noch große Herausforderungen, und beschreibt schließlich ein „Dilemma“: Einerseits würden an die Professionalität von NichtRegierungs-Organisationen (NROs), insbesondere in ihrer Eigenschaft als Gesprächs- und Projektpartner von Regierungen, mittlerweile immer höhere Anforderungen gestellt, ande- 
rerseits stelle sich aber die Frage, wie eine (weitere) Professionalisierung erfolgen solle, ohne dabei die dem Feminismus zugrundeliegenden radikalen Ideen aufzugeben und ohne dabei das bisherige Maß an Autonomie zu verlieren - in der Tat ein grundlegendes Problem, das allerdings auch anderen Bewegungen und Organisationen (jenseits des Feminismus in Lateinamerika) Schwierigkeiten bereitet hat und bereiten wird.

Der für den Rezensenten interessanteste Beitrag stammt aus der Feder von Jörg Faust (Universität Mainz, Institut für Politikwissenschaft). Er beschäftigt sich mit dem „Aufstieg und Fall der mexikanischen Autokratie“ während der 71-jährigen Herrschaft der „Partei der Institutionalisierten Revolution“ - PRI - , die nach längerer Agonie schließlich mit der im Juli 2000 erfolgten Wahl des konservativen Kandidaten Vicente Fox zum Staatspräsidenten endete. Der Autor verfolgt mit seiner Abhandlung das Ziel, die politische und wirtschaftliche Entwicklung Mexikos „,in ein institutionen-ökonomisches Modell autokratischer Herrschaft einzubetten“: Er charakterisiert die PRI als autokratisches Regime, das lange Zeit bis hin zum Ende der siebziger Jahre gesamtwirtschaftliches Wachstum gefördert habe, dann aber in eine Lage makroökonomischer Instabilität geraten sei, was zu einer allmählichen und langjährigen Phase der Machterosion geführt habe, und fragt sich, weshalb es zu gerade dieser Entwicklung gekommen ist. Auf dieser Basis entwickelt der Autor das o.g. Modell autokratischer Herrschaft, indem er einige typische, gleichsam gesetzmäßige Abläufe hervorhebt: Autokratien bräuchten Wachstum zur Befriedigung bestimmter erhöhter Finanzbedarfe wie etwa der Repressionskosten für den Überwachungsapparat, der Bedienung der Herrschenden aber nicht zuletzt auch der Versorgung der Masse der politisch ausgeschlossenen, damit diese sich nicht erhebe. Zur Deckung dieses erhöhten Finanzbedarfs griffen Autokratien auf das Instrument der Ressourcenmobilisierung (statt der eher unpopulären Produktivitätssteigerung) zurück, was sich in erhöhter Verschuldung, territorialer Expansion oder in einer Neigung zu riskanten Wachstumsstrategien äußern könne. Solange das Wachstum durch Ressourcenmobilisierung voranschreite, gebe es auch genügend Verteilungsmasse, damit sich die Mehrheit mit dem Regime arrangiere. Allerdings gerate die Autokratie irgendwann in eine „Modernisierungsfalle“, da das Wachstum einerseits weiterhin ungleich zu Gunsten der Eliten verteilt werde, andererseits die mit dem Wachstum verbundene Modernisierung zu mehr Emanzipation der Benachteiligten führe, was bei diesen steigende Unzufriedenheit hervorrufe, die in offene, systemkritische Opposition münde. Komme es schließlich dazu, dass nicht mehr genügend Ressourcen für das notwendige Wachstum mobilisiert werden könnten, so führe diese Wirtschaftskrise zur Bedrohung der politischen Stabilität. Die ursprünglich für die Autokratie systemstabilisierenden Klientelismus- und Korruptionsstrukturen kehrten sich nun gegen das System, da dieses seinen „Rentenverpflichtungen“ nicht mehr nachkommen könne. Der immer deutlicher werdende Erosionsprozess verselbständige sich und führe schließlich zum Ende des autokratischen Regimes.

Der Autor erreicht sein Ziel, die während der PRI-Herrschaft erfolgte wirtschaftliche und politische Entwicklung Mexikos in das o.g. Autokratiemodell ,einzubetten“, auf gelungene Weise, indem er die für die einzelnen Phasen des 71-jährigen PRI-Regimes wesentlichen 
wirtschaftlichen und politischen Tendenzen beschreibt und diese den jeweils einschlägigen Stationen seines Autokratiemodells zuordnet. Dadurch entsteht der Eindruck eines fast zwangsläufig vorgegebenen Ablaufs dieses langen Kapitels der mexikanischen Geschichte. Inwieweit das ,institutionen-ökonomische Autokratiemodell“ des Autors verallgemeinerungsfähig ist, soll und kann hier nicht erwogen werden; jedenfalls handelt es sich um einen spannenden und in seinem Gedankengang gut nachvollziehbaren Beitrag, der sich für Mexiko-Interessierte wie für „Autokratie-Forscher“ gleichermaßen attraktiv ausnimmt.

Insgesamt bleibt festzuhalten: Die bewährte Kombination aus dokumentarischer Faktenaufarbeitung einerseits und der vertiefenden Analyse andererseits hat im Lateinamerika-Jahrbuch 2001 erneut reichhaltige Frucht getragen.

Frank Niemeyer, Hamburg

\section{Sabine Kurtenbach (Hrsg.)}

\section{Kolumbien zwischen Gewalteskalation und Friedenssuche}

Möglichkeiten und Grenzen der Einflussnahme externer Akteure

Schriftenreihe des Instituts für Iberoamerika-Kunde, Hamburg, Band 54

Vervuert-Verlag, Frankfurt/Main, 2001, 253 S., € 24,80

Der vorliegende Reader thematisiert einen Konflikt, der - von der Weltöffentlichkeit nur sporadisch bemerkt - als ältester seiner Art in Lateinamerika den Staat Kolumbien seit mehr als einem halben Jahrhundert immer wieder zwingt, praktisch ohne Gewaltmonopol am Abgrund seiner Inneren Souveränität zu manövrieren. Bestandsaufnahme und mögliche Zukunftsperspektiven waren im Sommer 2000 Gegenstand einer interdisziplinär besetzten Fachtagung im Hamburger Institut für Iberoamerika-Kunde. Die Referate dieser Tagung sind hier in überarbeiteter Form zusammengetragen. Dass sie den Diskussionsstand von zwei Jahren vor den letzten Parlaments- (10. März 2002) und Präsidentenwahlen (26. Mai 2002 mit dem 46,8 \%-Erdrutsch-Wahlsieg des Ex-Liberalen Álvaro Uribe Vélez) wiedergeben, beeinträchtigt den Informationswert der Lektüre nicht: Es geht um Hintergrundwissen zum Verständnis dessen, was dieses Land noch auf absehbare Zeit wird durchzumachen haben.

Der umsichtig redigierte Band präsentiert sich unter drei Leitaspekten: Ursachen, Struktur wie auch interne Beteiligte - einschließlich der Opfer des Konflikts - werden in den drei Beiträgen des ersten Teils (,Dimensionen der Gewalt“) vorgestellt. Der „Friedenssuche“ widmen sich die sechs Beiträge des zweiten Teils, ehe der dritte Teil mit fünf Beiträgen den Blick auf „Externe Akteure“ und ihre gegenwärtige und womöglich auch künftige Rolle lenkt. 
Den ersten Teil eröffnet der Beitrag des an der Universität Bogotá und derzeit in Paris lehrenden Ökonomen Jaime Zuluaga Nieto „Das kolumbianische Labyrinth: Annäherung an die Dynamik von Krieg und Frieden“. Das mit der „Annäherung“ stimmt: Auf dem verfügbaren Raum war nicht mehr zu leisten als eine grob konturierende Skizzierung des komplexen, meist zulasten der Bevölkerung auf fatale Weise sich auswirkenden Kräfteparallelogramms aus einerseits offiziellen Sicherheitsorganen (Polizei, Streitkräfte) und ihren inoffiziellen paramilitärischen Pendants (AUC - Autodefensas Unidas de Colombia) sowie andererseits der guerilla mit ihren Hauptakteuren FARC (Fuerzas Armadas Revolucionarias de Colombia) und ELN (Ejército de Liberación Nacional). Im Bemühen um Unbefangenheit will es dem Außenstehenden mitunter schwer fallen, der ungeachtet etlicher Symptome organisierter Kriminalität gängigen „Kriegs“-Rhetorik zu folgen, in welcher sich die Beteiligten eingerichtet haben und der sich augenscheinlich auch manche Beobachter nicht verschließen mögen.

„Aktionsräume von Guerilla und paramilitärischen Organisationen: Regionale und bevölkerungsgeographische Auswirkungen“ überschreibt Günter Mertins, Geographie-Professor in Marburg, seinen eindrucksvollen Beitrag über die Konsequenzen regional paralysierter Staatlichkeit: Neben den erschütternden Hintergründen für die zwischen 1985 und 2000 von der Penetration durch Guerilla und Paramilitärs (Stichworte: Vertreibung, Zwangsrekrutierungen, Kindersoldaten, Schutzgelderpressung, Narcoguerilla) mehr als zwei Millionen um ihre Heimat gebrachten „Desplazados“ aufschlussreich das eingefügte statistische Material: Beispielsweise gingen von den allein in den ersten sieben Monaten des Jahres 2000 in Kolumbien registrierten insgesamt 155 Angriffen auf Polizeiorgane 10 auf das Konto der AUC, 114 verübten die FARC, 23 die ELN, der Rest entfiel auf kleinere guerilla-Organisationen.

Geschichte ist inzwischen das Phänomen „Farclandia“, jener der FARC 1998 in ihrer Heimatregion im Süden für die Dauer von „Friedensverhandlungen“ zugestandenen entmilitarisierten Zone von 3,7 \% der Landesfläche, mit 42.000 qkm so groß wie die Schweiz. Mit diesem „Staat im Staate“, den die FARC offenkundig keineswegs zur Probe aufs Exempel für eine Umsetzung ihrer offiziellen Ideale von Demokratie und Legitimität zu nutzen bestrebt war, Schluss zu machen, sah noch Präsident Andrés Pastrana Arango sich im März 2002 kurz vor dem Ende seiner Regierungszeit gezwungen.

Clarita Müller-Plantenberg, die an der Gesamthochschule Kassel Soziologie lehrt, schildert in ihrem Beitrag „Destabilisierung der Regionen und gesellschaftliche Gegenstrategien" die prekäre Lage, in welche insbesondere indigene Völker und Gemeinschaften, von denen es in Kolumbien an die 85 gibt, geraten durch wirtschaftliche Großprojekte wie Holzabbau, Wasserkraftanlagen, Bodenschätze-Prospektion. Von daher in struktureller Opposition zur Regierung, erweisen sie sich im Radius der Gewalt nicht weniger gefährdet durch den namentlich von der guerilla favorisierten Drogenanbau und seine Konsequenzen (Flächenverbrauch, illegaler Flugbetrieb, Munitionsfabriken, ökologische Schäden durch staatliche Herbizid-Sprühaktionen). Obwohl staatlicher Beistand, etwa in Form positiver 
Gerichtsentscheidungen, in seiner Realisierung an nahe Grenzen zu stoßen pflegt, fehlt es nicht an ermutigenden Gegenbeispielen.

Den zweiten Teil leitet der Augsburger Soziologe Peter Waldmann ein, den Lesern dieser Zeitschrift bekannt von seiner Herbert-Krüger-Gedächtnisvorlesung (VRÜ 31 [1998], 427 ff.). Klar faktengestützt spricht er unter dem Thema „Friedensgespräche und Gewalteindämmung" den substaatlichen Konfliktkräften den Willen ab, ihre profitable Symbiose mit den Rauschgiftkartellen gegen ihre ungewisse Macht-Perspektive in einem befriedeten Rechtsstaat einzutauschen. Vor dem Hintergrund chronischer struktureller Autoritätsunfähigkeit des kolumbianischen Staates nach innen hält der Autor für leidlich gangbar nur die Konsens-Variante in Form unbeirrter und beharrlicher „Friedensgespräche“, auch um den Preis, dass diese trotz demokratischen Legitimitätsvorsprungs der Regierung notgedrungen auf Augenhöhe geführt werden müssen. Immerhin biete sich hier ein autoritätsubstituierendes Forum für befriedende Einflüsse namentlich externer Akteure.

In letzterem sieht Sabine Kurtenbach, Herausgeberin und als Politologin am Hamburger Institut für Iberoamerika-Kunde Gastgeberin der Tagung, einen von mehreren Unterschieden zu früheren Friedensprozessen. Deren Impulsen für die Gegenwart spürt sie nach mit ihrer Frage „Kann Kolumbien aus seiner Geschichte lernen? Die aktuelle Bedeutung des Friedensprozesses der Regierung Betancur 1982-1986“. Ihr Fazit, dass Friedensprozesse, die sich durch in den eigenen Reihen nicht durchsetzbare Abmachungen selber überfordern und auf diese Weise scheitern, die Gewaltspirale eher zu beschleunigen drohen als zu bremsen versprechen, könnte mit einem womöglich absehbaren Paradigmenwechsel unter der neuen Regierung Uribe durchaus Besorgnis weckende Aktualität gewinnen.

Die Frankfurter Politologin Linda Helfrich-Bernal befasst sich mit „Demokratisierung und Rechtsstaatlichkeit als Mittel der Befriedung - das Beispiel der Guerillaorganisation M-19“. Was hier auf den ersten Blick wie eine Verwechslung von Methode und Resultat anmutet, löst die Autorin auf zu einer kenntnisreichen und im Ergebnis überzeugenden Analyse der M-19-Integration ab 1990 in den politischen Prozess des Landes. Sie illustriert zugleich, wie ernsthafte staatsseitige Reformkompetenz als Nagelprobe fungieren kann für die zivilgesellschaftliche Politikwilligkeit einer waffenführenden Konfliktpartei.

Den Beitrag von Hans R. Blumenthal, Leiter der Fundación Friedrich-Ebert de Colombia in Bogotá, „Der Friedensprozess: „Verhandlungen im Kriege“ sollte man im direkten Anschluss an die Einführung von Zuluaga Nieto lesen. Was am Thema nach Clausewitz klingt, liest sich auch im Inhalt so: Wiederholt den Klassiker zitierend, liefert Blumenthal auf der kompletten Folie der beteiligten Akteure eine leidenschaftslos umfassend fundierte Analyse aller wesentlichen - einschließlich historischer - Konflikt-Façetten. Sie mündet mit Blick auf die Friedensprozesse in El Salvador und Guatemala in die eher resignierende Perspektive, dass nicht uno actu die multiple Gewalt in Kolumbien zu enden ansteht, vielmehr das Land an ,(...) seinen vielen Plagen, deren zentrale - neben Gewalt Drogenhandel, Korruption, Straflosigkeit und Ungleichheit sind, (...) noch erheblich länger $\mathrm{zu}$ leiden und zu arbeiten" haben werde. 
Pedro E. Valenzuela, Politologe an der kolumbianischen Universidad Javeriana, gibt in seinem von der Herausgeberin souverän übersetzten Beitrag „Bewaffneter Konflikt, Neutralität und Gewaltlosigkeit. Erfahrungen an der Basis“ einen wertvollen Einblick in die bisherige Bilanz von Erfolgen wie Risiken der Bestrebungen im wesentlichen ländlicher indigener Gemeinschaften, strikte Neutralität zu praktizieren, indem sie sich der Kooperation mit bewaffneten Akteuren jedweder Couleur möglichst konsequent verweigern. Vor allem heißt dies, mutig, überwiegend sogar todesverachtend, zwischen den Fronten - die Streitkräfte mitgemeint $-\mathrm{zu}$ agieren.

Vice versa die entsprechenden „Berufsrisiken“ von kritischen Journalisten und Wissenschaftlern in den Blick nimmt der Hannoveraner Soziologe Klaus Meschkat: „Kolumbien und die Intellektuellen“. Hier öffnet sich eine Perspektive, die angesichts allein zwischen 1980 und 2000 insgesamt 149 ermordeter Journalisten und etlicher Wissenschaftsangehöriger das Maß an Schutz, welches Verfassungen und deren Wirklichkeiten hierzulande in Europa garantieren, eindrücklich als kaum überschätzbar wertvolles Gut zu würdigen nahe legt.

Den Dritten Teil, betreffend die externen Akteure, eröffnet Georg Boomgarden, Lateinamerika-Beauftragter im Auswärtigen Amt und schon im Contadora-Prozess an der Vorbereitung der beiden San José-Konferenzen 1984 und 1985 beteiligt, unter dem Thema „Prioritäten der deutschen Außenpolitik und der Konflikt in Kolumbien“. In persönlicher - nicht offizieller - Lageanalyse positioniert er die Bemühungen und die Handlungsmöglichkeiten der Bundesrepublik dort, wo dem Friedenswillen ernsthaft verantwortungsbereiter Kräfte der Zivilgesellschaft unter die Arme zu greifen ist. Ebenfalls aus deutscher Sicht befasst sich der am Deutschen Institut für Entwicklungspolitik in Bonn tätige Politologe Christian von Haldenwang mit den „Möglichkeiten und Grenzen der Einflussnahme durch Entwicklungszusammenarbeit“. Sie werden an den Zielen Stärkung zivilgesellschaftlicher Institutionen und Prozesse unter den wesentlichen Leitaspekten Sicherheit, Integration und Wirtschaftsstimulierung festgemacht

Thomas Fischer, Erlanger Historiker, rückt mit seiner Frage „Durch mehr Krieg zum Frieden? Die USA und der Plan Colombia“ dem Problem einer quasi janusköpfigen Befriedungs-Strategie zu Leibe: Der Ende 1999 von der Regierung Pastrana gemeinsam mit den USA erarbeitete „Plan für den Frieden, die Prosperität und die Stärkung des Staates“ sollte mit insgesamt 7,5 Mrd. US-Dollar an nationalen und internationalen Finanzhilfen (vor allem der USA) das Land einerseits durch institutionelle und soziale Stabilisierung des zivilen Sektors befriedungsfähig machen, andererseits aber auch mit militärischen Mitteln das Drogengeschäft nachhaltig bekämpfen im Interesse der Hauptkonsumentenländer, allen voran der USA. Mit den Regierungswechseln in den USA und in Kolumbien und seit sich die ebenfalls um Mithilfe angegangene EU der Unterstützung dieses Plans wegen seiner militärischen Komponente verweigerte, ist es still geworden um ihn.

Rainer Huhle, Politologe am Nürnberger Dokumentations- und Informationszentrum für Menschenrechte mit dreijähriger praktischer Erfahrung im 1997 eröffneten Verbindungsbüro des UNHCHR in Bogotá, reflektiert in seinem Bericht „Das Kolumbienbüro des UN- 
Hochkommissars für Menschenrechte - eine kritische Bilanz" Erfolge und Rückschläge institutionalisierter praktischer Menschenrechtsarbeit (Beobachtung, Beratung, Berichterstattung) in denkbar problematischem Umfeld.

Was wäre ein Kolumbien-Reader ohne einen Beitrag von Gerhard Drekonja-Kornat, seit Jahrzehnten ausgewiesener Landeskenner und als Politologe in Wien lehrend. In seinem spannenden, den Band beschließenden Beitrag „Contadora für Kolumbien?“ ruft er in wohltuend plastischer Sprache die wesentlichen Leitlinien und strukturellen Voraussetzungen der intra-lateinamerikanischen, seinerzeit von Mexiko, Venezuela, Panama und Kolumbien getragenen Contadora-Friedensinitiative für Zentralamerika in den frühen Achtzigern des vergangenen Jahrhunderts in Erinnerung und - winkt im Ergebnis ab: Angesichts der seither regional- wie geopolitisch gründlich veränderten Rahmenbedingungen sei in Lateinamerika derzeit kein Träger für einen solchen individuellen oder kollektiven externen Friedensimpuls verfügbar, zumal den Schlüssel zum Erfolg ohnehin Washington in Händen halte, das sich aber von der fundamentalistischen, auf das DrogenThema fixierten Definition seiner regionalen Sicherheitsinteressen nicht werde lösen wollen. Wieweit diese vor dem 11. September 2001 verfasste Analyse gegenwärtig noch dunkler einzufärben wäre, hat die Herausgeberin inzwischen an anderer Stelle beschrieben (Nord-Süd-Aktuell, April 2002, S. 103 ff.).

„Ist der kolumbianische Staat überhaupt noch zu retten?“ fragte am 08. März 2002 Josef Oehrlein in der FAZ. Bücher wie das vorliegende können dazu beitragen, diese Frage zu bejahen, gewiss nicht heute und auch nicht übermorgen - irgendwann aber schon.

Karl-Andreas Hernekamp, Hamburg

Krystian Complak (Hrsg.)

Europa Wschodnia - Ameryka Lacińska

Pozycja jednostki i system rządu

Europa del Este - América Latina

Estatuto del individuo y sistema de gobierno

Wydawnictwo Uniwersytetu Wrocławskiego, Wrocław, 2002, 426 S., 35,00 zł

Südamerika und Osteuropa: Einen Dialog ihrer Staatsrechtler herbeizuführen war das Ziel der Professoren Calogero Pizzolo, Buenos Aires und Krystian Compak, Breslau (Wrocław), als sie das „Erste Verfassungsrechtlertreffen Lateinamerikas und Osteuropas“ organisierten. Diese Konferenz, veranstaltet im September 2001 in Breslau, sollte an frühere polnisch-mexikanische Begegnungen anknüpfen; Anliegen der Organisatoren war jetzt aber, mit dem weiteren Teilnehmerkreis einen wesentlich größeren geographischen Raum zu erfassen. In dem vorliegenden Band, der die Referate der Tagung zusammenführt, finden 
sich aus Europa Texte lettischer, litauischer, polnischer, weißrussischer, tschechischer, slowakischer, ungarischer, ukrainischer, moldawischer, rumänischer und bulgarischer Verfassungsjuristen; Lateinamerika wird repräsentiert von Verfassungsrechtlern aus Costa Rica, Kolumbien, Venezuela, Peru, Brasilien und Argentinien. Die amerikanischen Beiträge sind durchgehend auf Spanisch verfasst, die europäischen auf Polnisch, Englisch, Russisch und Französisch. Vorangestellt ist eine Einführung und ausführliche polnische Zusammenfassung von Krystian Complak zu jedem Aufsatz; Zusammenfassungen der slawischsprachigen Texte auf Spanisch oder Englisch fehlen.

Die Tagung hatte als erste ihrer Art zunächst zwei allgemeine Themenstellungen: Einen Schwerpunkt bildeten die Regierungssysteme, der andere lag im Bereich der verfassungsmäßig gewährleisteten Grundrechte und ihrem Schutz. Der Band ist dementsprechend in zwei Teile gegliedert, was indes wegen häufiger Überschneidungen in den Beiträgen nicht immer überzeugt.

Ein Vergleich zwischen lateinamerikanischen und mittel- und osteuropäischen Lösungen zum jetzigen Zeitpunkt erscheint deshalb reizvoll, weil beiderorts in den letzten Jahren der Aufbau stabiler demokratischer Systeme die Aufgabe war. Fraglich ist allerdings, inwieweit generalisierende Betrachtungen hinsichtlich dieser Großregionen möglich sind. Scheint die Entwicklung in den lateinamerikanischen Ländern wegen ihrer historischen, sprachlichen und religiösen Gemeinsamkeiten auf den ersten Blick noch eher erfassbar zu sein, so verfügt in Mittel- und Osteuropa jedes Land über seine eigene Staats- und Rechtssprache, hinzu kommen neben nationalen auch religiöse Abgrenzungskriterien. Um so verdienstvoller ist deshalb der Artikel von Jerzy Jaskiernia, Abgeordneter der Demokratischen Linksallianz und Justizminister, ehe er seinen jetzigen Lehrstuhl an der Jan-KochanowskiAkademie übernahm. Über einen engen Begriff seines Titels „Regierungssysteme der Staaten Mittel- und Osteuropas“ hinausgehend gibt er (auf polnisch) einen Gesamtüberblick über die Verfassungstransformation in den einzelnen Staaten nach dem Zusammenbruch des Kommunismus, den der „Völkerherbst“ des Jahres 1989 und die Auflösung der UdSSR 1991 markieren. Seitdem muss jeder der von der sowjetischen Vorherrschaft befreiten Staaten seinen eigenen Weg der Systemtransformation finden und sich dabei entscheiden, ob und wie weit er sich der in Westeuropa begonnenen Integration des Kontinents anschließen will. Der Autor betont zu Recht die wichtige Rolle des Europarates bei der Durchsetzung von Demokratie, Rechtsstaatlichkeit und Menschenrechtsschutz in der Region. An dem Verhältnis eines Staates zu dieser Organisation habe man stets den Stand seiner politischen Reformen ablesen können. Der erreichte Erfolg zeige sich an der Aufnahme der meisten Staaten (mit Einschränkungen im Falle Russlands und der Ukraine und der fortwährenden Ausnahme Weißrusslands). Die nächste Probe sei nun der Beitritt zur Europäischen Union, der weitere Anforderungen an die Funktionsfähigkeit des Staatsapparats stelle. Im Mittelpunkt der Darstellung steht neben dem Wahlrecht, den verbreiteten Problemen bei der Stabilisierung der Parteienlandschaft und der Justizorganisation die Untersuchung der Regierungssysteme im engeren Sinne. Als konträre Lösungen benennt Jaskiernia einerseits Tschechien und Ungarn als parlamentarische Systeme mit von den 
Parlamenten gewählten Staatsoberhäuptern und andererseits Weißrussland, Russland und die Ukraine als Staaten mit besonders ,starken“ Präsidenten. Dabei sähen jedoch auch dort die Verfassungen dem Präsidenten nicht zugeordnete Regierungen vor, deren Bestand vom Vertrauen der Parlamentsmehrheit abhänge. Aus der Übersicht ergibt sich, dass die meisten Staaten sich für die Direktwahl ihres Staatsoberhauptes entschieden haben und ihm mehr Kompetenzen zugestehen, als es z.B. Deutschland tut, diese aber nicht an die des französischen Präsidenten heranreichen. Die im Verhältnis zum „klassischen“ parlamentarischen System stärkere Stellung des Präsidenten folge vielfach aus der damit verbundenen Erwartung größerer politischer Stabilität. Ob dies berechtigt ist, untersucht der Autor nicht. Dagegen scheint zu sprechen, dass gerade Tschechien und Ungarn in den neunziger Jahren besonders stabile politische Verhältnisse aufwiesen.

Eines der neuen „semipräsidentiellen“ Mischsysteme behandelt der französischsprachige Beitrag von Genoveva Vrabie, die an der Universität von Iaçi lehrt, über Rumänien. Sie stellt das in ihrem Land mit der Verfassung von 1991 gewählte Modell vor und setzt es in einen vergleichenden Kontext. Ihren Blick richtet sie jedoch nicht auf die anderen Transformationsländer in unmittelbarer Nachbarschaft mit einer ähnlichen organisierten „Doppelspitze“ der Exekutive, obwohl ein Vergleich unter Berücksichtigung der Verfassungspraxis der letzten Jahre von großem Interesse für eine Bewertung der gemischten Systeme wäre. Abschließend finden sich Überlegungen zum Verhältnis Präsident - Regierung Parlament bei unterschiedlichen Mehrheitsverhältnissen an Hand der Erfahrungen mit der neuen Verfassung.

Lateinamerikas Regierungssysteme schildern überblicksartig Paulo Napoleão Nogueira da Silva, Professor in Toledo/Acatuba, aus brasilianischer und Daniel Alberto Sabsay, Professor in Buenos Aires, aus argentinischer Sicht. Beide beurteilen die dortigen Präsidialsysteme negativ. Das US-Vorbild sei nicht richtig umgesetzt worden bzw. für die Region gar nicht geeignet. Die checks and balances würden dort nicht greifen, statt dessen kennzeichne den lateinamerikanischen Präsidentialismus eine enorme Machtkonzentration in den Händen des Staatschefs. Sabsay benennt als Mitursache strukturelle Unterschiede wie namentlich die typische Schwäche von Parlamentarismus und Föderalismus. Da z.B. in Argentinien die nationalen Abgeordneten sich stärker ihren Parteien und weniger ihren Provinzen zugeordnet fühlten, seien die parteipolitischen Trennlinien viel schärfer als im US-Kongress. Anders als dort könnten in Lateinamerika Präsidenten nur äußerst schlecht mit gegenläufigen parlamentarischen Mehrheiten kooperieren. Deshalb werde vielfach versucht, an ihnen vorbei mittels Dekreten zu regieren. Sabsay beschreibt Reformversuche des Regierungssystems in Argentinien, Eloy Espinosa-Saldaña Barrera, der an der Universidad Católica in Lima lehrt, solche in Peru. Unter anderem seien in beiden Ländern mit dem Ziel einer Separierung von Regierungs- und Präsidialfunktionen u.a. die Ämter des Jefe de Gabinete bzw. des Presidente del Consejo de Ministros geschaffen worden. Deren Aufgabe beschränke sich jedoch im wesentlichen auf die Organisation der Regierungstätigkeit; eine selbstständige rechtliche und politische Position gegenüber den Präsidenten hätten sie nicht. Letztere blieben in beiden Ländern nicht nur Staats- sondern auch Regie- 
rungschefs. Damit könnten die Reformen keine spürbaren Veränderungen bewirken. Ebenfalls pessimistisch sieht der argentinische Verfassungsjurist Alberto Antonio Spota die Versuche zur Verbesserung des Föderalismus in seinem Lande - und zwar sowohl hinsichtlich der Modifikationen, die den Zentralstaat stärken sollen, als auch derer, die zu mehr regionaler und lokaler Autonomie führen müssten. An der Universität Corrientes (Argentinien) lehrt Héctor J. Zimerman, der die Probleme der Demokratie in Lateinamerika aus politologischer Sicht analysiert; er stellt die Regierungsweise des „klassischen“ der des heutigen „Neopopulismus“ hinsichtlich Stils der Herrschaftsausübung und der Wirtschaftspolitik einander gegenüber.

Die Situation in Lateinamerika und in Mittel- und Osteuropa nach dieser Lektüre im Kontext betrachtet, kommt in den europäischen gemischten Systemen trotz Stärkung der Präsidenten die entscheidende Rolle den von den Parlamenten abhängigen Regierungen zu. In den Staaten Lateinamerikas besteht hingegen regelmäßig kein eigenständiges exekutives Machtzentrum neben den Präsidenten. Solange davon keine radikale Abkehr stattfinden soll, sondern nur an eine Evolution des als problematisch angesehenen Präsidentialismus gedacht ist, würde der Weg wohl am ehesten in Richtung der russischen und ukrainischen Varianten gehen. Wie diese sich bewähren, muss sich noch zeigen; Michail Bajmuratov von der Nationalen Rechtsakademie in Odessa beschreibt in seinem Beitrag über das ukrainische Modell nur dessen Grundzüge (auf Polnisch).

Der den Grundrechten gewidmete Teil des Bandes beginnt mit Lateinamerika: Nestor Pedro Sagüés von der Katholischen Universität Rosario/Argentinien behandelt in seinem Überblick anhand der einschlägigen Verfassungstexte die jeweiligen Staatsmodelle, referiert die Typologie der jeweils gewährleisteten Grundrechte und die Prozeduren zu ihrem Schutz. Mehrheitlich - so sein Ergebnis - sei das Verfassungsleitbild der soziale Rechtsstaat. Interessant ist auch, vom Umgang in den einzelnen Staaten mit den sog. derechos imposibles zu lesen, also solchen Grundrechtsgarantien, die der Staat vor allem aus materiellen Gründen - entgegen seinem Versprechen in der Verfassung - nicht erfüllen kann. Nicht unerwähnt bleibt in diesem Beitrag zudem der allgemein oft enorme Unterschied zwischen Verfassungstext und -wirklichkeit. Der Hauptteil widmet sich dem Rechtsschutz: Ausführlich werden die vielfältigen nationalen Organisationsformen gerichtlichen Grundrechtsschutzes Modellen zugeordnet, Vor- und Nachtteile der Lösungen beleuchtet und die unterschiedlichen Verfahren beschrieben. Dabei resümiert der Autor, eine wünschenswerte Annäherung der Systeme werde durch die herrschende ,terminologische Anarchie“ erschwert: Gleiche Rechtsinstitute würden unterschiedlich benannt; umgekehrt könnten unterschiedliche Institute in verschiedenen Ländern die gleiche Bezeichnung haben.

Für einen wichtigen Teilbereich mehr Klarheit schaffen will Calogero Pizzolo mit seinem Beitrag „Las fórmulas sobre amparo en el derecho constitucional latinoamericano”. Nach seiner zusammenfassenden Definition fallen unter den Begriff des amparo Mechanismen, die dem Rechtsschutz des Bürgers vor dem Staat dienen. Der Verfasser beschränkt sich auf den Schutz der durch die Verfassung gewährleisteten Rechte und liefert so mit Blick auf die gesamte Region eine vergleichende Studie zu den Ausprägungen der Verfassungsbe- 
schwerde. Behandelt werden Prozessvoraussetzungen, Verfahrensgang, Verfahrensziel und die rechtliche Einordnung des Instituts. Der Freiheitsgarantie des Habéas Corpus aus der Perspektive seines Heimatlandes Costa Rica sind die kurzen Ausführungen von Máximo Sequeira Alemán von der Universität San José, gewidmet. Weiterhin finden sich noch Länderberichte zu den Grundrechten und ihrem normativen Schutz von Hernán Alejandro Olano García, Universität La Sabana/Kolumbien und von Adrián Vargas Benavides, Richter am Verfassungssenat des Obersten Gerichtshofs in Costa Rica zur Verfassungsgerichtsbarkeit.

Hervorgehoben seinen noch einige dogmatische Untersuchungen zu Einzelthemen: Ana Elvira Araujo García von der Zentraluniversität Caracas analysiert für Venezuela rechtsvergleichend das prozessuale Erfordernis der Verletzung eines subjektiven Rechtes für die Beschreitung des Verwaltungs- / bzw. Verfassungsrechtsweges in ihrem Land. Susana Graciela Cayuso von der Universität Buenos Aires verfolgt die legislativen und richterlichen Ausformungen des Gleichheitsgrundsatzes im argentinischen Recht. Ihr Kollege Jorge Alejandro Amaya widmet sich dem Datenschutz und dessen Bezug zu Verbraucher und Markt: Er beleuchtet die Situation in Argentinien vergleichend vor dem lateinamerikanischen und westeuropäischen Hintergrund.

Die europäischen Beiträge über den Schutz der Grundrechte sind großenteils Länderberichte. So behandeln Nóra Chronowski, Assistentin an der Universität Pecz und ihr dortiger Kollege József Petrétei für Ungarn und Ján Drgonec, ehemals Richter am Verfassungsgerichtshof für die Slowakei (beide Aufsätze in englischer Sprache) sowie Anita Uschatska, Richterin am Verfassungsgerichtshof der Republik Lettland in Riga (auf russisch), ausführlich die jeweilige Rechtslage einschließlich der mittlerweile von den Gerichten entwickelten Dogmatik. Weiter hervorzuheben ist die kürzere polnischsprachige Darstellung von Jan Filip, der an der Masaryk-Universität in Brünn lehrt und auf die historisch bedingte tschechische Besonderheit einer neben der Verfassung stehenden eigenen Grundrechtscharta eingeht. Er skizziert im weiteren unter Verzicht auf allen Ländern Gemeinsames das System in seinem Heimatland; eingearbeitete statistische Daten geben Ausschluss über die Inanspruchnahme der Rechtsschutzinstrumente durch die Bürger. Beachtung verdient in diesem Zusammenhang der russischsprachige Aufsatz von Nadeschda Semenowa, Vorsitzende der Liga für Konstitutionalismus und Rechtskultur in Kiew, über die Ukraine. Dieser Staat muss schon auf Grund kaum vorhandener eigener rechtsstaatlicher Traditionen, an die angeknüpft werden könnte, und der besonders starken Durchdringung mit dem sowjetischen Modell besonders große Anstrengungen bei der Einführung rechtsstaatlicher Institutionen unternehmen. In weiteren kürzeren Beiträgen skizziert die Rechtslage in Bulgarien Simeon Bozanow, Professor an der Universität Ruse (auf Russisch), unternimmt es Alvydas Pumputis, Professor an der an der Litauischen Universität Wilna, nach der Auflistung wichtiger Jahreszahlen für die Etablierung der Grundrechte in seinem Land diese allgemein von den menschlichen Bedürfnissen herzuleiten und behandelt Valeriu Zubco von der Moldawischen Humanistischen Universität in Kischinau die konkrete Normenkontrolle in Moldawien (beide auf Englisch). 
Als Beispiel ähnlicher Mechanismen zum Rechtsschutz der Bürger wird die Institution des Ombudsmanns thematisiert - bei Christian A. Cao, Universität Buenos Aires, für Argentinien und dazu im Vergleich mit Polen bei Adam Zieliński, Universität Warschau, (auf Polnisch). Zieliński betont die Überwindung der Diktatur und den Aufbau der Demokratie als gemeinsame Aufgaben in beiden Ländern; er lässt indes unerwähnt, dass das Amt des polnischen Bürgerrechtsbeauftragten noch zu kommunistischen Zeiten geschaffen wurde, um formal mehr Rechtsstaatlichkeit zu gewährleisten, allerdings ohne den Bürgern die Möglichkeit zu geben, mittels Verfassungsbeschwerde den Schutz ihrer Grundrechte selbst einzuklagen.

Von westlichen Vorbildern sich kritisch abzugrenzen versucht lediglich Sergej Kalinin vom Lehrstuhl für Staats- und Rechtstheorie sowie Geschichte an der Staatlichen Weißrussischen Universität in Minsk in seinem ins Polnische übersetzten Beitrag. Als Gegenmodell zur ideologischen Anlehnung an die ,atheistische“ westliche Menschenrechtskonzeption sieht er eine - weiter reichende - Konzeption auf der Grundlage des christlich-orthodoxen Bekenntnisses. Seine Referenzquellen sind Fundstellen des Evangeliums, das offenbar manche der in den letzten Jahrzehnten gültigen „Klassiker“ abgelöst hat.

Als Fazit der meisten Beiträge zum Grundrechtsschutz kann festgehalten werden: Auch wenn die Verfassungswirklichkeit in den einzelnen Staaten Lateinamerikas und MittelOsteuropas sich noch recht unterschiedlich darstellt und oft von der praktischen Wirksamkeit der Schutzmechanismen gar nicht erst die Rede ist, so haben sich dank der Veränderungen in den letzten Jahre die verfassungsmäßigen Leitbilder und Institute in den Grundzügen bemerkenswert einander angeglichen. Verfassungsbeschwerde und konkrete Normenkontrolle sind mittlerweile weit verbreitet, der subjektivrechtliche Gehalt von Grundrechten und ihre unmittelbare Geltung fast überall anerkannt.

Abschließend sei im Hinblick auf die künftige Entwicklung in den beiden Regionen der jeweilige supranationale Kontext noch einmal hervorgehoben: Für die mittel- und osteuropäischen Staaten, die die Integration mit der Westhälfte des Kontinents anstreben, werden auch im Verfassungsrecht die westeuropäischen Lösungen der Hauptbezugspunkt bleiben. In Lateinamerika hingegen beachtet man bei der Verfassungsfortbildung zwar die westeuropäischen Entwicklungen; sie sind dort jedoch nicht im gleichen Maße Vorbild wie für die europäischen Reformstaaten. Auch ist eine weitgehende Integration dort bisher nicht dauerhaft gelungen bzw. auch nur geplant. Andererseits sind in Amerika wie in Europa die Grundwerte von Demokratie und Rechtsstaatlichkeit fast überall Gemeingut. In ihrem Rahmen haben auch die europäischen Transformationsländer einen großen Spielraum bei der Gestaltung ihrer Verfassungsordnung. Da in beiden Regionen noch vieles im Fluss und weniger verfestigt als z.B. in Westeuropa ist, wäre eine Fortsetzung des Dialogs gerechtfertigt und wünschenswert.

Ulrich Ernst, Berlin, z.Zt. Krakau 


\section{Erik Jayme (Hrsg.)}

\section{Das Recht der lusophonen Länder}

Tagungsreferate, Rechtsprechung, Gutachten

Nomos Verlagsgesellschaft, Baden-Baden, 2000, 251 S., $€ 51,00$

Dieser Sammelband umfaßt zunächst die Erträge der Jahrestagungen 1997 (Porto) und 1998 (Halle) der Deutsch-Lusitanischen Juristenvereinigung zivilrechtlichen und zivilprozessualen Inhalts. Hinzu kommen weitere, z.T. bereits anderweitig veröffentlichte Beiträge, Gutachten vor allem zum portugiesischen Erbrecht sowie eine umfangreiche „Portugalrechtsbibliographie (1955-Sept. 1999)“ einschließlich kurz referierter Rechtsprechung (S. 201-239).

Für die Leser dieser Zeitschrift dürften zwei Beiträge von Interesse sein. Die Studie „Einführung in das brasilianische Umweltrecht" von Antônio Herman V. Benjamin (S. 105139) ist ohne Zweifel Glanzstück dieses Sammelbandes. Der Autor schildert die historische Entwicklung des Umweltrechts bis hin zu der ersten juristischen Wahrnehmung eines erforderlichen Umweltschutzes etwa bezüglich der Industrieverschmutzung (Lei do Zoneamento Industrial nas Áreas Críticas de Poluição von 1980) und der Pflanzenschutzmittel (Lei de Agritóxicos von 1998). Als ein eigens durch die Verfassung zu schützendes Gut und damit einen neuen Rang erhielt die Umwelt durch ihren juristischen Schutz in der Bundesverfassung (Constituição Federal), Art. $225 \S 1$ Abs. 1: Erhalt und Wiederherstellung von essentiellen ökologischen Prozessen, d.h. die ,sozial-umweltrechtliche Funktion des Eigentums" wurde eingeführt. Die daraus abgeleiteten expliziten als auch impliziten Rechte und Pflichten bezüglich des Umweltschutzes und die verfassungsrechtlichen Kompetenzen werden behandelt. Wichtigstes umweltrechtliches Instrument zur effektiven Verwirklichung des Umweltschutzes ist das Gesetz Nr. 6.938/81 - Lei da Política Nacional do Meio Ambiente. Erst mit diesem Gesetz beginnt der Umweltschutz als solcher in Brasilien (S. 108). Die Einzelheiten werden daher eingehend vorgestellt, ergänzt um die Darstellung der anderen, segmentarischen Umweltgesetze. Einen nachhaltigen Schutz der Umwelt durch Umsetzung der Gesetze soll die Ação civil pública - Lei Nr. 7.347/85 - (,öffentliche Zivilklage") bringen, die auch privatrechtlichen Nicht-Regierungsorganisationen offen steht und von diesen bereits häufig angewendet sein sollen (Zahlen nennt der Verfasser leider nicht). Die daneben bestehende umweltrechtliche Popularklage - ação popular ambiental - soll keine nennenswerte Auswirkung gehabt haben.

In einem kurzen, jedoch inhaltlich dichten Beitrag (S. 157-165) beschäftigt sich Rui Manuel Moura Ramos mit der Gemeinsamen Portugiesisch-Chinesischen Erklärung über Macau vom 13. April 1987 aus völkerrechtlicher Sicht. Moura Ramos referiert die wesentlichen Punkte dieses von ihm als völkerrechtlicher Vertrag, nämlich Instrument der Gebietsabtretung und der Schaffung und Regelung der Übergangsperiode (bis 20. Dezember 1999), qualifizierten Systems. Er weist darauf hin, daß die Gemeinsame Erklärung nach dem auch hier (neben Hongkong) verwirklichten Grundsatz „ein Land, zwei Systeme“ ein Sonderverwaltungsgebiet Macau der VR China begründet mit einem hohen Maß an Auto- 
nomie. Die Festschreibung mittels der Vorschriften des Völkervertragsrechts (S. 164) gebe diesem Gebiet eine andere Rechtsgrundlage als nur nach dem Gesetz der VR China vom 31. März 1993. Wenn dazu der portugiesische Ausdruck ,lei básica“ verwendet wird, so nur als Übersetzung des englischen „Basic law“ für die parallele Situation in Hongkong. Unterhalb der Ebene des von der Zentralen Volksregierung ernannten Regierungschefs existieren Organe, die die legislativen, exekutiven und judikativen Befugnisse Macaus eigenständig ausüben, besetzt durch Bewohner des Sonderverwaltungsgebietes Macau, Art. 24, 25, 27-41 der lei básica. Dabei bleiben die Rechte auf politische Teilhabe den ständigen Bewohnern des Territoriums vorbehalten.

Der Verfasser sieht - verhalten optimistisch - in der Gemeinsamen Erklärung einen usus modernus des Völkerrechts, der dieses nicht nur als typisches Werkzeug zur Lösung von zwischenstaatlichen Streitigkeiten und als Verfahren zur Abstimmung der sich in diesem Bereich gegenüberstehenden Interessen von Rechtssubjekten begreift. In diesem usus könnte ein Ansatz für eine Ausgestaltung der internationalen Gemeinschaft gesehen werden, auf den zumindest in bestimmten außergewöhnlichen Situationen zurückgegriffen werden könnte.

Gerhard Scheffler, Hamburg

\section{Karin Fiedler}

\section{Wirtschaftsethik in China am Fallbeispiel von Shanghaier Protestanten}

Zwischen Marx und Mammon

Mitteilungen des Instituts für Asienkunde, Hamburg, Nr. 327

Institut für Asienkunde, Hamburg, 2000, 234 S., € 19,00 (broschiert)

Sebastian Heilmann

\section{Die Politik der Wirtschaftsreformen in China und Rußland}

Mitteilungen des Instituts für Asienkunde, Hamburg, Nr. 317

Institut für Asienkunde, Hamburg, 2000, 310 S., € 25,00 (broschiert)

\section{Matthias Fronius}

Die Ursachen des taiwanesischen Wirtschaftswunders

Eine systemische Betrachtung

Schriften zur Wirtschafts- und Sozialgeschichte, Band 68

Duncker \& Humblot Verlag, Berlin, 2001, 245 S., € 69,00 (broschiert)

Der sprunghafte wirtschaftliche Aufschwung in der Volksrepublik China nach den verlustreichen Irrungen des maoistischen Caesarismus provozierte zahlreiche Erklärungsversuche, die sich in die - oft auch in praktischer Politik hochkontroversen - Debatten reihen lassen, 
welche seit Marxens analytischem Versuch über eine ,asiatische Produktionsweise“ erst den Gründen des sozio-ökonomischen Rückstands der fernöstlichen Region gegenüber dem neuzeitlichen Westen und in unseren Tagen - etwa unter dem Rubrum ,asiatischer Werte“ - den Antrieben von deren vielfach rasantem Aufholen nachzuspüren trachteten.

Anfang der neunziger Jahre veranstalteten in Shanghai die Weltbank und die Stadtregierung, die gerade ihr Projekt der Entwicklungszone Pudong auf den Weg gebracht hatte, ein Symposium zur Entwicklungsstrategie, auf dem eine Leuchte der US-amerikanischen Ostasienforschung in gut einstudiertem Chinesisch der überwiegend örtlichen Zuhörerschaft vielleicht verschmitzt - erläuterte, wie die drei kleinen Wirtschafts-Tiger Taiwan, Hongkong und Singapur zum Erfolg gekommen seien. Der Professor aus dem fernen Neuengland verkündete im Kern, dem ersten hätten die japanischen Kolonialherren von 1895 bis 1945 die Bedeutung von A-r-b-e-i-t vorbuchstabiert, den beiden anderen hätten die Briten beigebogen, was ,Ordnung' und ,Disziplin' heißen. Während der Redner unbekümmert fortfuhr, wurde es rasch still im Saal. Als der Mit-Vorsitzende, der damalige Bürgermeister und heutige Ministerpräsident Zhu Rongji, zu Fragen einlud, eilte ein Zuhörer zum Mikrophon im Parkett, begann, den Gastredner erregt zu attackieren, und war erst durch mehrfaches Zureden von Mitarbeitern des Bürgermeisters, der die gedeihliche Atmosphäre der Veranstaltung mit dem wichtigen internationalen Geldgeber wahren wollte, zum Ablassen zu bewegen. Der Gelehrte aus Massachusetts dürfte sich an jenem Vormittag in Shanghai kaum Freunde erworben haben. Die Erklärung moderner Entwicklungserfolge in den „konfuzianischen“ Gesellschaften Ostasiens rührt ebenso an kollektive Nerven wie das Brüten über den früheren katastrophalen Rückstand, der China - anders als Japan - nicht erlaubte, den Kanonenbooten der ,goldhaarigen und grünäugigen Barbaren“ Einhalt zu gebieten.

Die Dissertation von Katrin Fiedler über Shanghaier Protestanten im China der postmaoistischen „Reform und Öffnung“ erörtert mithin ein schwieriges und kontroverses Thema. Neben einleitender Darstellung der mit Max Weber begonnenen Erklärungsansätze zu ,protestantische[r] Ethik und de[m] Geist des Kapitalismus“" sowie einem Überblick zur zahlreichen modernen chinesischen Literatur zu gegenwärtigen wirtschaftsethischen Fragen bilden Befragungen, Gruppengespräche und Beobachtungen der Autorin unter Shanghaier Protestanten den empirischen Kern der Arbeit. Mission und Einfluß christlicher Religion in China, nach dem frühen kulturellen rayonnement der Jesuiten im kaiserlichen China seit dem Opiumkrieg von 1840-42 zunehmend im Gefolge abendländischer Marineflaggen, konsularischer Hoheitszeichen und europäischer Händler gediehen, wurden trotz mancher segensreicher Wirkungen im Reich der Mitte dort oft als aggressive geistliche Globalisierung wahrgenommen. Zumal angesichts des gespannten Verhältnisses des atheistischen kommunistischen Regimes zur Religion wird eine Rolle von Christen als „Salz der Erde“, aus der Volks-Chinas moderne Wirtschaft wachsen soll, daher besonders akribisch zu erweisen sein. $\mathrm{Ob}$ die 28 Partner von Einzelgesprächen in der Untersuchung - wiewohl offenbar der im soziologischen Handwerk empfohlenen Reichweite entsprechend (S. 59) vom Platz der Christen unter den über zwölf Millionen Einwohnern von Chinas erster 
Wirtschaftsmetropole genug Aufschluß geben können, erscheint dem Rezensenten umso zweifelhafter, als mindestens zwölf von diesen 28 kraft der beruflichen Stellung als untergeordnetes Personal einzustufen sein dürften, dem in Shanghais politischer Ökonomie Einfluß kaum zukommen dürfte. Die Arbeit beschreibt so weniger eine meßbare Teilhabe von Christen oder deren Glauben am Reformprozeß in der VR China, gibt aber mit ihrer Darstellung der wirtschaftsethischen Diskussion, deren veröffentlichte Teile die Autorin dankenswert gründlich gesichtet hat, einen aufschlußreichen Einblick in die verwickelten Debatten, zu denen sich eine kommunistische Einparteienregierung genötigt sieht, die aus vielfachen Beweggründen (,dem Volke zu dienen“, dem Erhalt eigener Legitimität durch greifbare Erfolge, patriotischer Restauration von Chinas gloire) eine ordnungspolitische Kehrtwende vollziehen muß, für die das Rüstzeug des nach außen unverändert behaupteten staatskommunistischen Machtkatechismus sich nicht mehr glaubhaft dienstbar machen läßt. Nicht immer überzeugen die in der Arbeit vorgestellten Kontraste, die in protestantischer Ablehnung von Luxus und Hingabe an mühsame Arbeit eine Triebfeder individuellen und kollektiven Fortkommens sehen, die ,weder im klassischen chinesischen noch im sozialistischen Denken existier[e]“ (S 33). Schon Meister Kong dürfte hier widersprochen haben ${ }^{1}$, zahllose strebsame Chinesen Hong Kongs, Taiwans oder Südostasiens, deren kleingewerbetreibende Familien zusammenlegen, um Sprößlingen das Studium in Australien oder Nordamerika zu erlauben, gleichermaßen. Die Frage nach der aktiven Zutat, die einem gesellschaftlichen Gemisch modernisierende Kraft verleiht, bleibt offen; eine Schlüsselstellung ,protestantischer Wirtschaftsethik“ auf Chinas Reformwegen hat diese dennoch interessante Arbeit nicht dargetan. Am Rande: Das Wortspiel zur „Bibelitis“ (shengjingbing) - vgl. S. 224 - dürfte auf das viel ähnlichere, umgangssprachliche shenjingbing (Geisteskrankheit) zielen, nicht auf das förmlichere, gleichbedeutende jingshenbing.

Der totalitäre Kommunismus in den mächtigsten Staaten des schon seit langem gespaltenen „sozialistischen Lagers“, China und der Sowjetunion, zerfiel auf äußerlich dramatisch verschiedene Weise. Dies lieferte ein Paar faszinierender Studienobjekte für Voraussetzungen und Strategien des Übergangs aus Planwirtschaften zu marktförmigen und - in der Sicht einiger damit sogar unvermeidlich verbunden - demokratischen Ordnungen. Die wirtschaftlichen Erfolge post-maoistischer Reformen verkehrten zum Teil dabei das frühere Verhältnis, in dem Peking den ,großen Bruder Sowjetunion“ (Sulian lao dage) in den fünfziger Jahren als Vorbild genommen hatte. Bedienstete russischer Auslandsvertretungen in China etwa sahen zeitweilig einer Rückversetzung nach Moskau ohne Begeisterung entgegen, waren doch dort die Lebensverhältnisse karger als damals in Peking oder Shanghai. Tiefer Skepsis ausländischer politischer Beobachter und Kaufleute zur Zukunft Rußlands stand, steht, - auch in den „Dollarvoten“ der Anleger sichtbar - sonniges Vertrauen in die Aussichten gegenüber, die ein Engagement in „Rot-China“ verspreche. Die machtvgl. die Übersetzung von D.C. Lau, Confucius, The Analects, The Chinese University Press, Hongkong, 1979. 
politische Bedeutung beider Länder verlieh zugleich den Rezepten von Politikberatern aus den Denkfabriken des Westens den Nimbus strategischen Gewichts, ähnlich kriegsentscheidendem Vermögen, feindlichen Funkverkehr zu entschlüsseln - könnte doch ein ,richtiger“ Reformkurs mit darüber bestimmen, ob sich diese riesigen Länder friedlich zu internationalen Partnern entwickeln oder - in eruptiven Veränderungen - politische Trümmer auf die übrige Welt schleudern würden.

Professor Heilmanns Habilitationsschrift gebührt das Verdienst, die Reformverläufe in China und der Sowjetunion bzw. danach der Russischen Föderation im Rahmen der einschlägigen politik- und wirtschaftswissenschaftlichen Forschung sorgsam verglichen zu haben. Auch wenn russisch- oder chinesischsprachiges Schrifttum zugunsten vornehmlich englischsprachiger Literatur kaum berücksichtigt wurde, zeichnet die Studie ein eindrucksvolles Bild der unterschiedlichen Wege zur Neuordnung in beiden Ländern. Trotz zahlreicher gemeinsamer Ausgangsbedingungen - Einparteienherrschaft und Planwirtschaft unterschieden sich beide dennoch in für Neuanfänge wichtigen Punkten. So erlaubte die wesentlich geringere planwirtschaftliche Durchdringung und Industrialisierung der chinesischen Landwirtschaft, aber auch der Industrie, in Peking leichter, dezentralisiertes Wirtschaften wieder zu beleben. Zugleich blieb in China die KPCh - anders als die aus der Macht verstoßene KPdSU in der späten Sowjetunion und späteren Russischen Föderation leninistisch straff organisierte Ordnungskraft, die Rahmenbedingungen unternehmensgebundenen Wirtschaftens eher zu setzen und erhalten vermochte als diverse Machthaber in Moskau. Schließlich war in China gewerbliche Wirtschaft, die - oft mit ausländischem Kapital - neben der staatlichen neu zugelassen wurde, eine weit wichtigere Wohlstandsquelle als der Energieträgersektor (Öl, Gas) im Nachbarland. Tragfähige Bedingungen des Wirtschaftens in herstellenden Branchen wurden so zur Voraussetzung auch korrupter Teilhabe der kommunistischen Nomenklatur Chinas am wirtschaftlichen Aufstieg, während in Rußland die natürlichen Ressourcen durch plündernden Zugriff alter Machteliten versilbert werden konnten, ohne daß dafür tiefgreifender marktwirtschaftlicher Umbau zu betreiben war. Beide Länder stehen in ähnlichen Faktorengeflechten, in denen sie aber hinsichtlich einzelner Entwicklungen - Herausbildung demokratischer Institutionen in Rußland, Anreize zu gesamtwirtschaftlich förderlichem inländischen Wettbewerb in China - unterschiedlich plaziert sind. In China und Rußland gleichermaßen bleibt das oft beschworene „ebene Spielfeld“ für wirtschaftliche Akteure rechtlich und praktisch erst zu schaffen, bleiben tragfähige Individualrechte erst anzuerkennen und zu schützen. Ebenfalls sind in beiden Staaten korrupte und - mehr in Rußland als in China - sogar gewalt-kriminelle Zugriffe auf Staat und Gesellschaft während der Phase ungeordneten Wandels zu einem Grad gewachsen, der ihre Beseitigung ohne neuen totalitären Rückfall nicht rasch erlauben wird. Eine ,universell gültige Anleitung für die erfolgreiche Bewältigung des Transformationsprozesses zur Marktwirtschaft“ kann aber, resümiert die Studie, ,die wirtschafts- und politikwissenschaftliche Theorie (...) nicht an die Hand geben“ (S. 283). Nur am Ende (S. 273) streift der Autor einen perspektivischen Unterschied: In Rußland wurde grosso modo 
der Abschied von stalinistischem Kommunismus mit Ausnahme bislang randständiger chauvinistischer Strömungen seitens der führenden Personen als Annäherung an euroatlantische Ordnungsmuster begriffen. In China meint die Zielformel vom „Sozialismus mit chinesischen Charakteristika“ erklärtermaßen den Erhalt autokratischer Herrschaft, was zumal durch die ,vier Grundprinzipien““2 im Vorspruch zur Verfassung von 1982 als normativer Bannfluch gegen jede Forderung nach demokratischem Aufbruch konkretisiert ist und für China - im Gegensatz zu Rußland - den künftigen Weg zu freiheitlicher Modernität wenigstens in offizieller Dogmatik als einen Konfliktkurs mit der etablierten Macht vorzeichnet, den Chinas WTO-Beitritt nur verschärfen kann.

Chinas staatliche Einigung datiert geläufig auf das Jahr 221 vor Christus, als innerhalb der machtlos verdämmernden Zhou-Dynastie im Machtkampf der sieben „streitenden Reiche“ das Königreich der Qin seine Rivalen unterwarf und mit der eigenen gleichnamigen Dynastie im Reich der Mitte wieder eine wirksame Zentralgewalt schuf. Spätere Spaltungen führten unter verschiedensten Vorzeichen doch immer wieder zu einheitlicher Herrschaft. Teilstaaten im zerfallenen Reich - oder gegen fremde Eroberer hinhaltend sich behauptende Reste früherer Dynastien, wie die südliche Ming-Dynastie gegenüber den von Norden eingefallenen Mandschus - waren indes sämtlich nach gleichem politischen Muster gestaltet und bewahrten noch in blutiger Sezession den politischen Gleichklang der ,, chinesischen Welt “33. Das Hin und Her dynastischer Trennung, Eroberung oder Wiedervereinigung wurde von der Teilung Chinas nach dem festländischen Bürgerkrieg von 1945-49 zwischen Mao Zedongs Kommunisten und Chiang Kai-sheks Nationalisten erstmals - wenngleich um Jahrzehnte verzögert - strukturell durchbrochen: Der Aufstieg Taiwans, eines Altwassers chinesischer Besiedlung, vom portugiesisch-holländischen Zankapfel und letzter Bastion Ming-zeitlicher Getreuer zum Gegenstand der mission civilisatrice des ostasiatische Sonnenplätze erheischenden Japans, zum Vorposten von US-amerikanischem containment gegenüber Rot-China und nun zum Wirtschaftswunder-Territorium mit ungewissem völkerrechtlichen Status markiert spätestens seit dem friedlichen Machtwechsel zum Präsidenten Chen Shuibian im Jahr 2000 außerdem eine grundlegende, demokratische Alternative zum säkularen autokratischen Regiment chinesischer Tradition. Ähnlich wie die

Nämlich: „Festhalten am sozialistischen Weg, der volksdemokratischen Diktatur, der Führung durch die KPCh und dem Marxismus-Leninismus sowie dem Mao-Zedong-Denken“; ein Tetralog, den die autoritative Enzyklopädie Cihai von 1999 unverändert zum „Born des Staatsbaus Chinas“ (Zhongguo de liguo zhi ben) erhebt. Zum ideologischen Gewicht des Cihai vgl. Li Chunping, Cihai jishi [Chronik des Cihai], Cishu chubanshe, Shanghai, 2000. Als Versuch einer Generalkritik an der ideologischen Fortentwicklung durch Jiang Zemin vgl. Bao Tong, Yisi he weisheng zhi ji, zai ping san ge daibiao [Zwischen schon Totem und noch Ungeborenem; eine erneute Kritik der drei Vertretungen], ZHENG MING [Hongkong] 2002 (September), 29 ff.

„Le monde chinois“, gängiger Terminus der französischsprachigen Sinologie, kennzeichnet verbal - wiewohl gegenüber dem chinesischen tianxia (all-under-Heaven) reduktionistisch - handlich jenen Inbegriff ideologischer Einheit von allem chinesisch vorgestellten imperium, dem sich auch Abtrünnige jeweiliger Dynastien offen oder doch schlüssig verpflichtet gaben. 
Auflösung der Sowjetunion liefert der Vorstoß Taiwans in die vorderen Reihen der Weltwirtschaft polit-ökonomischen Theoretikern einen empirischen - wenngleich grätenreichen - Leckerbissen: Taiwans Erfolgsgeschichte seit 1949 erweckt Interesse als mögliches Vorbild für eine aufholend sich entwickelnde „Dritte Welt“ ebenso wie als eine Vorform der Zukunft des festländischen Chinas und damit als mögliches Ziel des ungewissen Wandels, dem sich die Führung der VR China seit dem Ende des Mao-Régimes verschrieben will.

Die Dissertation von Matthias Fronius bietet einen gut lesbaren Überblick zur Vorgeschichte der modernen taiwanischen Eigenständigkeit seit 1949 und insbesondere zu den hauptsächlichen Erklärungsversuchen für den spektakulären Weg der Ilha formosa vom öden Eiland zum hochtechnologischen Wachstumspol. Einleitend beschreibt der Autor die Entwicklung Taiwans seit dessen Abtretung an Japan im Jahr 1895 bis in unsere Tage. Anschließend werden die gängigen theoretischen Erklärungskonzepte - das ,neo-klassische" mit dem Akzent auf freiem Außenhandel und staatlicher Zurückhaltung im Wirtschaftsleben, das "revisionistische" mit dem Akzent auf staatlicher Lenkung, das „,synkretistische“ der Weltbank und das Asien-wissenschaftlich geprägte „,kulturalistische“ - vorgestellt und auf ihre Erkenntniserträge hin geprüft. In einem eigenen - „systemischen“ Ansatz versucht der Verfasser schließlich, die von den anderen Ansätzen zu isolierter Wirkungsmacht erhobenen Faktoren der taiwanischen Entwicklung in einen gemeinsamen Zusammenhang zu stellen. Die ausschließlich westlich-sprachige Quellen verwertende Arbeit leidet kaum unter dem Ausschluß chinesisch- oder japanisch-sprachiger Literatur ${ }^{4}$ und demonstriert zugleich die Schwierigkeiten, den Entwicklungsprozeß Taiwans im Nachhinein befriedigend zu erklären, geschweige denn aus ihm anleitenden Wert für künftige Politik zu gewinnen. Die Gesamtschau der Faktoren, die der Autor selbst versucht, erinnert aber an andere Orte, wo sich kleine politische Gebilde unter hohem Druck und Ungewißheit ums Überleben sorgen mußten. Israel, Singapur, Süd-Korea oder Hongkong mußten alle zur Zukunft schmale Pfade gehen, an deren Rändern Abgründe gähnten. Das Bewußtsein, bei Strafe der Vernichtung nicht grob fehltreten zu dürfen, veranlaßte die auch nach ihrer Flucht nach Taiwan weiter vom Festland bedrohte, durchaus autokratische Nationalistische Partei (KMT) Chiang Kai-sheks zu später politischer Klugheit, die ihr, als sie sich der Macht auf dem Festland noch gewiß wähnte, nicht gekommen war. Verdienst der KMT ist es, die Potentiale chinesischer Lernkultur, infrastruktureller Hinterlassenschaft Japans und geo-strategischer Bedeutung der Insel für die USA zu einer Politik genutzt zu haben, die Taiwans Bewohnern erlaubte, ihre Ressourcen erfolgreich zu mobilisieren. Angesichts der Herrschaftsansprüche Pekings bleibt jedoch Gratwanderung zwischen

Kleine Irrtümer, wie die Annahme, „die Schriftsprachen Japans und Taiwans [seien] ähnlich genug, so daß ein Chinese problemlos eine japanische Zeitung lesen kann“ (S. 168), fallen für die Schlußfolgerungen der Arbeit nicht ins Gewicht. Dennoch ist lange nach dem Ende der japanischen Kolonialzeit auch unter Jüngeren das Interesse an japanischer Sprache und Kultur in der Tat groß geblieben. 
Wohlstand und Untergang für die politischen Nachfolger Chiangs auf absehbare Zeit unentrinnbare Erblast.

Wolfgang Kessler, Berlin

\section{Florian Kutz}

\section{Amnestie für politische Straftäter in Südafrika}

Von der Sharpeville-Amnestie bis zu den Verfahren der Wahrheits- und

Versöhnungskommission

Berlin Verlag Arno Spitz, Berlin, 2001, 322 S., € 45,00

Politische Systemwechsel können in einer Gesellschaft (und bei jedem einzelnen Betroffenen) ganz unterschiedliche Reaktionen hervorrufen. Das Spektrum reicht dabei von Vergeltung gegenüber ehemaligen Machthabern bis hin zu Versöhnung mit den für schwere Menschenrechtsverletzungen Verantwortlichen. In den meisten Fällen wird sich der nach einem staatlichen Neuanfang eingeschlagene Kurs jedoch - abhängig von der Intensität des vorangegangenen Unrechts und der neuen politischen, wirtschaftlichen und militärischen Kräfteverteilung - irgendwo zwischen diesen beiden extremen Koordinaten einpendeln. Die beunruhigenden Entwicklungen in Simbabwe zeigen außerdem, dass die Schatten der Vergangenheit innenpolitische Konflikte auch nach vielen Jahren erneut aufbrechen lassen und die Grundlagen eines zunächst gefundenen Konsenses erschüttern können.

Ein Beispiel für eine solche Gesellschaft im Übergang ist Südafrika. Das Land, jahrzehntelang von einer weißen Minderheit dominiert, versucht seit 1990, die politischen, wirtschaftlichen, gesellschaftlichen und sozialen Folgen von Apartheid zu bewältigen. Dabei deuten die erst kürzlich von Opfern der Rassentrennung in New York geltend gemachten Schadensersatzforderungen darauf hin, dass auch die Südafrikaner trotz aller Fortschritte die ,historic bridge between the past of a deeply divided society characterised by strife, conflict, untold suffering and injustice, and a future founded on the recognition of human rights, democracy and peaceful co-existence“ (so der Abschnitt über „National Unity and Reconciliation“ in der Verfassung von 1993) noch nicht sicher überschritten haben. Die in Berlin vorgelegte Arbeit von Florian Kutz wirft ein Licht auf diesen Prozess. Der Verfasser lenkt den Blick dabei auf einen der schmerzhaftesten Kompromisse, den das vom charismatischen Nelson Mandela propagierte und von der großen Mehrheit seiner Mitbürger angenommene Ziel der gesellschaftlichen Versöhnung vielen unmittelbar Betroffenen abverlangt hat und weiterhin abverlangen wird: Amnestie für politische Straftäter im Austausch gegen die Aufklärung ihrer Verbrechen.

Von der südafrikanischen Übergangsverfassung als einer der tragenden Pfeiler gesellschaftlicher Versöhnung (reconciliation) und staatlichen Wiederaufbaus (reconstruction) 
besonders hervorgehoben, ist Amnestie neben strafrechtlicher Verfolgung und materieller Wiedergutmachung ein wichtiges, zugleich aber auch problematisches Instrument im Umgang mit den Verbrechen der Vergangenheit. Der Verfasser, durch eine zweijährige Mitarbeit bei der Wahrheits- und Versöhnungskommission in Kapstadt unmittelbar mit deren Amnestiepraxis vertraut, nähert sich dem Thema, indem er einen kurzen Überblick über die Optionen gibt, die einer Gesellschaft im Übergang von Diktatur zu Demokratie bei der Aufarbeitung von Menschenrechtsverletzungen offen stehen. Ein kurzer geschichtlicher Abriss des Landes macht dabei deutlich, dass der Systemwechsel in Südafrika in die Kategorie einer verhandelten Machtübergabe fällt. Dies erklärt nicht nur viele Besonderheiten der neuen Verfassungsordnung, so etwa den Zusatz über „National Unity and Reconciliation“; die von Dion Basson als ,revolution negotiated between the oppressor and the oppressed" bezeichneten Reformen hatten in Südafrika auch wesentlichen Einfluss auf die Ausgestaltung und Umsetzung des Amnestiegesetzes von 1995. Dieses Gesetz war Grundlage für die Arbeit der südafrikanischen Truth and Reconciliation Commission und des von Mitgliedern der Kommission gebildeten Amnestieausschusses.

Schon die beiden Straffreiheitsgesetze von 1990 und 1992, von Kutz zusammen mit den seit Anfang der sechziger Jahre erlassenen Gesetzen zum Schutz von Funktionären und Bediensteten des Regimes im dritten Kapitel der Arbeit dargestellt, müssen vor dem Hintergrund dieser besonderen Kräftekonstellation am Ende der weißen Alleinherrschaft interpretiert werden. Von Kritikern als „self amnesty“ der scheidenden NP-Regierung gegeißelt, zeigt der Verfasser anhand einer sorgfältigen Analyse der durchgeführten Verfahren, dass etwa der Further Indemnity Act von 1992 weit überwiegend Mitglieder oder Angehörige der Freiheitsbewegungen begünstigte. Amnestiert wurden dabei vor allem Mord, aber auch Vergewaltigung, Raub, Brandstiftung und Diebstahl, und obwohl der gesamte Prozess ausschließlich politische Straftäter begünstigen sollte, belegt die Untersuchung, dass die Gewährung von Straffreiheit bis 1994 stark von machtpolitischen Interessen dominiert wurde und trotz der Orientierung an den in Namibia entwickelten Nørgaard Principles und mehreren Abkommen zwischen Regierung und Opposition nachvollziehbare Maßstäbe in der Praxis vermissen ließ. Ohne eine weitgehende Amnestie für die Anhänger der Widerstandsbewegungen, so der Eindruck des Lesers nach diesem Abschnitt der Arbeit, wäre eine friedliche Entwicklung in dieser ersten kritischen Phase des Übergangs jedoch nicht möglich gewesen.

Mit Umsetzung der in der Übergangsverfassung angelegten Individualamnestie, durch den 1995 erlassenen Promotion of National Unity and Reconciliation Act in eine verfahrensund materiellrechtliche Form gegossen, wurde dieser schwierige Prozess fortgesetzt. Dabei macht schon eine einleitende Bemerkung des Verfassers deutlich, dass die Aufarbeitung der Vergangenheit auch weiterhin auf der Geschäftsgrundlage des politischen Kompromisses von 1993 erfolgen würde: „Weder war das neue, demokratische Südafrika bereit, Staatskriminelle und Straftäter aus den Reihen der Widerstandsbewegungen bedingungslos zu amnestieren, noch konnte die neue Ordnung die strafrechtliche Verfolgung dieser Personen durchsetzen." Bereits hier drängt sich allerdings die Frage auf, ob die neue staatliche Ord- 
nung unter diesen schwierigen Voraussetzungen überhaupt in der Lage war, die dem Amnestiegesetz zugrundeliegende Bedingung (Straffreiheit nur gegen Wahrheit) durchzusetzen und das angestrebte Ziel (Versöhnung) zu erreichen.

Die Arbeit beantwortet diese komplexe und für Südafrika auch heute noch offene Frage letztendlich nicht. Dies ist jedoch kein Vorwurf an den Verfasser. Ob das Amnestiegesetz wirklich Versöhnung erzielt hat, kann sicherlich nur mit Hilfe einer interdisziplinären Untersuchung ermittelt werden, die sich jenseits einer rein juristischen Perspektive insbesondere mit der Lage der Opfer und den weiteren Lebensläufen der Täter beschäftigen müsste. Außerdem ist Versöhnung, wie der Verfasser in seiner abschließenden Bewertung zutreffend anmerkt, ein langsamer Prozess; Erfolg oder Misserfolg der Amnestie werden daher erst in einigen Jahren sichtbar werden. Kutz konzentriert sich statt dessen auf die Rahmenbedingungen des Amnestieverfahrens, die materiellen Tatbestandsmerkmale der Amnestieregelungen und die Rechtsfolgen der ergangenen Amnestieentscheidungen. Dabei beschreibt er zahlreiche Verfahren vor dem Amnestieausschuss und stellt sie in den Kontext der einzelnen Gesetzesbestimmungen. In dieser Verknüpfung von Fällen und gesetzlichen Vorschriften liegt die Stärke der Untersuchung. Indem der Verfasser die Auslegung des Amnestiegesetzes (so etwa den Begriff der politischen Straftat) anhand von einzelnen Verfahren nachzeichnet, vermittelt er nicht nur Einblicke in die schwierige Arbeit des Ausschusses bei der Bewältigung der über 7.000 Anträge und leistet damit einen wertvollen Beitrag zur Ausgestaltung künftiger Amnestieverfahren; die beunruhigende Schilderung konkreter Fälle vermittelt dem Leser auch einen Eindruck der Aufarbeitungsleistung, die die so oft beschworene Formel von truth and reconciliation den Opfern und Angehörigen wirklich abverlangt. Zudem belegen die ausgewählten Sachverhalte die von Kutz mehrfach getroffene Feststellung, dass der Amnestieausschuss bei der Behandlung ähnlicher Anträge in bestimmten Punkten, so etwa bei der Definition der strafbaren Handlung, keine einheitliche Linie gefunden hat.

Die Heranziehung konkreter Sachverhalte birgt allerdings auch Probleme. So verwendet Kutz bei der Beschreibung der Amnestieanträge und in der Diskussion der Ausschussentscheidungen zwangsläufig deutsche strafrechtliche Kategorien wie etwa Mord, fahrlässige Tötung, Notwehr und Notwehrexzess, ohne dass geklärt wird, ob diese Begriffe im südafrikanischen Kontext einen vergleichbaren Bedeutungsinhalt aufweisen. Darüber hinaus wäre an einigen Stellen der Arbeit eine vertiefte Auseinandersetzung mit den Entscheidungen des Ausschusses wünschenswert gewesen. So wird etwa dessen Interpretation der amnestiefähigen politischen Straftat in sechs Teilaspekten systematisch nachvollzogen; die auf diese Weise gewonnenen Befunde werden aber teilweise nicht weiter problematisiert, obwohl das zusammengetragene Fallmaterial - etwa zur Notwendigkeit einer Anordnung oder Billigung der Tat durch den Staat oder eine politische Organisation - durchaus Anlass zu einer kritischen Würdigung des Gesetzes und seiner Umsetzung gegeben hätte. Schließlich zeigt die ausführliche Dokumentation des sogenannten St. James Massakers vom Juli 1993, dass die im Gesetz aufgeführten Amnestiekriterien - so etwa das politische Motiv des Täters, der Charakter der Tat (Auftrag oder eigener Tatentschluss) und die Auswahl des 
Opfers (politischer Gegner oder unbeteiligte Zivilperson) - durch den Amnestieausschuss bei der Bewertung einzelner Anträge nicht gleichmäßig berücksichtigt wurden. Das Ergebnis der Ausschussarbeit war vom Gesetzgeber als eine Gesamtabwägung von Tat und Täter konzipiert und gab dem Ausschuss im Einzelfall einen erheblichen Entscheidungsspielraum. Obwohl dieses Ermessen bei der Untersuchung einzelner Amnestiekriterien in der Arbeit angesprochen wird, hätte dieser sicherlich wichtige Aspekt vom Verfasser in einem separaten Abschnitt stärker beleuchtet werden können.

Insgesamt hat Kutz mit dieser Arbeit einen wichtigen Baustein zur Erforschung der Amnestieverfahren in Südafrika beigetragen. Das Modell der Individualamnestie wird dabei sowohl konzeptionell als auch in seiner praktischen Umsetzung mit gut abgewogenen Argumenten einer Generalamnestie oder der strafrechtlichen Verfolgung politischer Straftäter vorgezogen. Der Vorschlag, parallel zu einem internationalen Strafgerichtshof eine Wahrheitskommission nach südafrikanischem Vorbild einzurichten, erscheint zum heutigen Zeitpunkt allerdings verfrüht. Ungeachtet der mit der Einrichtung internationaler Gremien einhergehenden praktischen Schwierigkeiten ist das Experiment Individualamnestie in Südafrika auch nach Bescheidung aller Amnestieanträge noch nicht abgeschlossen. Die mit der Amnestie angestrebte Versöhnung hat den Opfern mit der Straffreiheit für die Täter und dem Schutz aller Beteiligten (Täter, Staat und Widerstandsbewegungen) vor jeglichen zivilen Schadensersatzansprüchen einen sehr hohen Preis abverlangt. Ob das beschränkte Beweisverfahren im Amnestieausschuss die angestrebte Gegenleistung - Wahrheit - so viel besser erzielen kann, als der ebenfalls auf Erkenntnis ausgerichtete Strafprozess, bedarf noch weiterer Klärung. Und ob das übergeordnete Ziel gesellschaftlicher Versöhnung in Südafrika tatsächlich erreicht wird, bleibt ebenfalls abzuwarten.

Jörg Fedtke, London

Christian P. Scherrer

\section{Genocide and Crisis in Central Africa}

Conflict Roots, Mass Violence, and Regional War

Praeger Publishers, Westport, CT / London, 410 S., £ 54,95

Der Völkermord in Ruanda ist nicht nur eine der größten menschlichen Katastrophen in der Zeit nach dem Zweiten Weltkrieg, der innerhalb von wenigen Wochen (April bis Juli 1994) über eine Million Menschenleben forderte und mehr als 2,4 Mio. Ruander zur Flucht ins benachbarte Ausland veranlasste, sondern auch ein schwarzes Kapitel in der Geschichte der Vereinten Nationen und der (westlichen) Welt. Der Genozid war kein überraschend aufgetretenes Ereignis, sondern kündigte sich mit mehrjähriger Vorlaufzeit an. Trotzdem war die Weltgemeinschaft nicht Willens und in der Lage, diese menschliche Tragödie zu verhin- 
dern. Im Gegenteil: Frankreich lieferte vor, während und nach dem Genozid Waffen und Munition nach Ruanda (S. XVI), und die westlichen Geber erhöhten ihre Entwicklungshilfe für das Regime, das den Völkermord vorbereitete.

Christian P. Scherrer beschäftigt sich seit Jahren mit der Krisenregion Zentralafrika und der Völkermordproblematik. Er hat verschiedene Studien zu diesem Themenbereich vorgelegt. ${ }^{1}$ Im Auftrag des Flüchtlingshilfswerks der Vereinten Nationen (UNHCR) war er an der Untersuchung des Völkermordes in Ruanda beteiligt.

Das vorliegende Buch leistet eine umfassende Analyse der politischen Entwicklung in Zentralafrika. Dieser über Ruanda hinausgehende Ansatz verdeutlicht die komplexen historischen, ethnischen, interessen- und machtpolitischen Zusammenhänge in der Region, vor deren Hintergrund der Völkermord von 1994 nur erklärt werden kann.

Scherrer analysiert im ersten Teil seiner Studie die Faktoren, die zum Genozid von 1994 geführt haben. Er verdeutlicht, dass der Völkermord über Jahre hinweg geplant und erkennbar vorbereitet wurde. Zunächst arbeitet er die historischen Dimensionen auf, die für den späteren Völkermord eine Rolle spielten. Er macht deutlich, dass erst die Kolonialmächte - und hier vor allem die Belgier (wie auch die katholische Kirche) - mit der Privilegierung der Tutsi die Wurzeln für die Konflikte zwischen Hutu und Tutsi setzten. Die Belgier führten 1926 ethnisch gekennzeichnete Personalausweise ein (S. 27). Die Rivalität zwischen Hutu und Tutsi zieht sich bis heute durch die Geschichte der Länder Ruanda und Burundi und bekam durch die vom Westen erzwungene und nicht ausreichend vorbereitete Einführung demokratischer Reformen den entscheidenden Anschub.

Im zweiten Teil des Buches untersucht der Autor den Völkermord in Ruanda von 1994. Er beschreibt die Vorbereitungen des Genozids und die unrühmliche Rolle der Kirchen, Geberländer und der UNO. Noch im Januar 1994 - drei Monate vor Beginn des Völkermordes -, nachdem die Genozidpläne auch der UN bekannt geworden waren, versicherte der offensichtlich völlig desinformierte Generalsekretär Boutros-Ghali dem ruandischen Staatschef Habyarimana - und damit dem Hauptverantwortlichen für diese Vorbereitungen - seines Vertrauens, die Angelegenheit zu lösen, was dieser auch tat, wenngleich freilich nicht im Sinne der UN. Er intensivierte seine Völkermordvorbereitungen (S. 92). Der staatlich organisierte Genozid wird ausführlich analysiert. Bemerkenswert ist, dass nicht nur staatliche, militärische und gesellschaftliche Akteure inklusive Vertreter der christlichen Kirchen an der systematischen Ermordung der Tutsi-Bevölkerung beteiligt waren, sondern auch die Hälfte der männlichen Hutu-Bauern, die im blinden Gehorsam an dem Gemetzel aktiv teilgenommen hatten (S. 115). Eine unrühmliche Rolle spielte der private Radiosender Radio Television Libre des Milles Collines (RTLM), der von Hutu-Extremisten betrieben wurde und offen zum Völkermord anstachelte. Die UN reduzierten nach dem Ausbruch des Genozids ihr Truppenkontingent statt es zu erhöhen und überließen damit das Land seinem Schicksal. Das war u.a. auf die ablehnende Haltung der USA 
zurückzuführen, die nach dem Somalia-Debakel kein weiteres militärisches Engagement in Afrika auf sich nehmen wollten. Ein Verhalten, für das sich Präsident Clinton auf einem Besuch in Kigali im April 1998 öffentlich entschuldigte.

Der dritte Teil der Studie beschäftigt sich mit dem Einfluss der Entwicklungshilfe auf die Vorbereitung und Durchführung des Völkermordes. Das Volumen der öffentlichen Entwicklungshilfe wurde trotz der unübersehbaren Genozidvorbereitungen und Menschenrechtsverletzungen zwischen 1990 und 1994 um 50 \% erhöht (S. 179). Kritisch wird auch die von nichtstaatlichen ausländischen Organisationen bereitgestellte Nothilfe für Flüchtlinge sowie die Entwicklungshilfepolitik und die Rolle internationaler Nichtregierungsorganisationen nach dem Genozid beleuchtet.

Im vierten Teil widmet sich Scherrer der Situation in Burundi sowie dem Krieg im Kongo, an dem mehrere Länder aktiv beteiligt sind. Der Autor versucht, das komplizierte Geflecht von Interessen der Nachbarstaaten und der einzelnen innerkongolesischen Interessen zu analysieren und mit einer großen Anzahl von Fakten zu untermauern. Er beklagt das geringe Interesse der UN, sich im Kongo mit einer zahlenmäßig adäquaten Friedenstruppe zu engagieren. Während 38.000 Soldaten im Auftrag der NATO im übersichtlichen Kosovo im Einsatz waren, wurden in den riesigen Kongo gerade 5.537 Soldaten entsandt (S. $303 \mathrm{f}$.).

Die detaillierte Chronologie über die Ereignisse der Krise in Zentralafrika von 1994-2001 bietet einen guten Überblick über die Ereignisse, obwohl die Spaltenanordnung etwas unübersichtlich ist. Im Schlusskapitel geht der Autor auch auf die juristische Aufarbeitung des ruandischen Genozids ein. Das Dilemma der Vergangenheitsaufarbeitung wird darin deutlich, dass das International Criminal Tribunal for Rwanda in drei Jahren gerade einmal 10 Verfahren abgeschlossen hatte, während insgesamt noch über 120.000 Verdächtige in Untersuchungshaft ihres Prozesses harren und viele Täter sich noch auf dem Gebiet der Demokratischen Republik Kongo versteckt halten. Diese sollen von Traditionalgerichten abgeurteilt werden, wobei leider nicht erklärt wird, wie diese gachacha-Gerichte funktionieren und wie ihre Richter gewählt werden (S. 367 f.).

Das Buch ist eine schonungslose Abrechnung mit dem Versagen der Vereinten Nationen und der westlichen Welt, allen voran Frankreich, die USA und die Schweiz, die die Tragödie von Ruanda durch ihr Verhalten befördert, zumindest nicht verhindert haben. Die persönliche Betroffenheit des Autors, der vor Ort recherchiert hat, zieht sich wie ein roter Faden durch das Buch. Es unterscheidet sich insofern von vielen anderen Veröffentlichungen zu dieser Thematik, die sich nur auf die wissenschaftliche Aufarbeitung und Analyse beziehen. Diese Eigenheit gerät der Studie nicht zum Nachteil, denn das Versagen der westlichen Welt, vor allem auf Grund von eklatanten Fehlperzeptionen und der Verfolgung eigener Interessen, ist bis heute beispiellos.

Die bisweilen ein wenig zu sehr ins Detail abgleitende Studie (vor allem die namentliche Nennung zahlreicher Personen erschwert die Lektüre und erinnert eher an ein Polizeiprotokoll) bietet eine gründliche, interessante und - sofern man das bei diesem ernsten Thema sagen darf - spannende Analyse mit der notwendigen Tiefenschärfe. Die schonungslose, 
aber sachlich begründete Kritik am Wirken der externen Akteure ist ein Aufruf, die Konfliktpräventionsmechanismen der UN so zu verändern, dass die Tragödie von Ruanda sich nicht wiederholt und vor allem, dass ein Völkermord wie in Ruanda nicht mit Hilfe von westlicher Entwicklungshilfe und Waffenlieferungen erst ermöglicht wird (S. 5). Dazu gehört die Aufnahme der Genozidprävention in die Anti-Genozid-Konvention der UN (S. 374). Den Fragen der Aufarbeitung des Genozids und der Verhinderung von Völkermord wird sich der Autor in einem bereits angekündigten zweiten Buch widmen. Man darf gespannt sein.

Heiko Meinhardt, Hamburg 